\title{
LLNL-TR-402609
}

LAWRENCE LIVERMORE NATIO N A L LABORATORY

\section{Fast Diagnostic For Electrical Breakdowns In Vacuum}

T. L. Houck, J. B. Javedani, D. A. Lahowe

March 28, 2008 
This document was prepared as an account of work sponsored by an agency of the United States government. Neither the United States government nor Lawrence Livermore National Security, LLC, nor any of their employees makes any warranty, expressed or implied, or assumes any legal liability or responsibility for the accuracy, completeness, or usefulness of any information, apparatus, product, or process disclosed, or represents that its use would not infringe privately owned rights. Reference herein to any specific commercial product, process, or service by trade name, trademark, manufacturer, or otherwise does not necessarily constitute or imply its endorsement, recommendation, or favoring by the United States government or Lawrence Livermore National Security, LLC. The views and opinions of authors expressed herein do not necessarily state or reflect those of the United States government or Lawrence Livermore National Security, LLC, and shall not be used for advertising or product endorsement purposes.

This work performed under the auspices of the U.S. Department of Energy by Lawrence Livermore National Laboratory under Contract DE-AC52-07NA27344. 


\title{
Fast Diagnostic For Electrical Breakdowns In Vacuum
}

\author{
T.L. Houck, J.B. Javedani, and D.A. Lahowe, \\ Lawrence Livermore National Laboratory, Livermore, CA
}

\begin{abstract}
The design of an inexpensive, small, high bandwidth diagnostic for the study of vacuum insulator flashover is described. The diagnostic is based on the principle of capacitive coupling and is commonly referred to as a D-dot probe due to its sensitivity to the changing of the electric displacement field. The principle challenge for the design proved to be meeting the required mechanical size for the application rather than bandwidth. An array of these probes was fabricated and used in an insulator test stand. Data from the test stand with detailed analysis is presented. A highlight of the application of the probes to the test stand was the ability to detect the charging of the insulator surface by UV illumination as a prelude to the insulator flashover. The abrupt change in the insulator's surface charge during the flashover was also detected.
\end{abstract}


Table of Contents

$\begin{array}{ll}\text { Abstract } & 1\end{array}$

Introduction 3

Theory of Operation 3

Electrical Design $\quad 4$

Probe Response - Scope signal compared to drive voltage 4

Practical Considerations $\quad 6$

$\begin{array}{ll}\text { Calculating Capacitances } & 7\end{array}$

$\begin{array}{lr}\text { Tolerances } & 8\end{array}$

Design Example $\quad 8$

Initial Estimates 9

Electrostatic Modeling: Capacitances $\quad 10$

Electrostatic Modeling: Errors $\quad 11$

$\begin{array}{ll}\text { Airline Calibration } & 12\end{array}$

Calibration Data versus Expected Response 12

Comments on Airline Calibration $\quad 13$

Parallel Electrode Testing 14

Raw Data 16

Correcting For Attenuation and Converting to Diode Voltage 17

Detailed Data Analysis $\quad 18$

Flashover Site 19

Detecting Surface Charge 20

Effect of Insulator Angle

Effect of Insulator Material 22

Higher Resolution Probe Comparison $\quad 24$

$\begin{array}{ll}\text { Summary } & 25\end{array}$

$\begin{array}{ll}\text { Acknowledgements } & 25\end{array}$

References $\quad 26$

$\begin{array}{ll}\text { Appendices } & 27\end{array}$

A. List of Symbols with Definitions $\quad 27$

B. Derivation of D-dot Probe Response to Specific $V_{D}$

C. Nominal Specifications of Commercial 50 Ohm, Copper Jacket, Coaxial Cable 30

D. Suggested Design Estimators $\quad 31$ 


\section{Introduction}

An important area of pulsed-power R\&D is high-voltage vacuum insulator breakdown, usually referred to as flashover due to the sudden avalanche of electrons across the insulator surface. This phenomenon is often the limiting factor in attaining the highest possible performance in pulsed-power devices. While several hypotheses attempt to explain the initiating mechanism(s), flashover is not well understood. Computational modeling is limited to establishing the electromagnetic field and radiation imposed on the insulator. The designer then makes adjustments to the configuration and/or materials until the modeling results are within safety margins based on empirical data. This design approach is believed to be overly conservative since the bulk dielectric strength of the insulating material can be an order of magnitude greater than the flashover limitation. However, without a quantitative understanding of the phenomenon this is the only approach to ensure reliability.

A major obstacle to gaining a quantitative understanding of insulator flashover is the time scale for the phenomenon. Typical vacuum electrode spacing is on the order of a centimeter depending on the voltage and the flashover will occur in a fraction of a nanosecond. Commercial oscilloscopes are available with sampling rates of multiple GHz. Thus, we require diagnostics with response times on the order of a few hundred picoseconds or faster. Equally important, the diagnostic must be compatible with the geometry of the experiment. In most applications and test stands, the insulator is located between parallel electrodes a few $\mathrm{cm}$ to tens of $\mathrm{cm}$ in transverse dimension and separated by distances of a few $\mathrm{mm}$ to a few $\mathrm{cm}$. Diagnostics that are located away from the electrode gap, e.g. on the power feeds, record a signal delayed and distorted by the intervening inductance and capacitance. These "stray" inductances and capacitances are normally very small, but for the extremely fast signals associated with flashover they act as filters removing the critical signal components.

Capacitive-coupled probes ${ }^{1}$ can be constructed with fast response times and in a broad range of geometries. These probes are often referred to as D-dots because they are sensitive to time variation in the electric displacement. The probes are often used as voltage probes when the locations of all free charges are known. In that case, the voltage (potential) producing the electric field can be calculated from the field detected at the probe. In this report the design of a simple, robust D-dot is described. A D-dot probe based on the design was constructed and used in a high-voltage, vacuum insulator test stand. The performance of this probe is presented.

\section{Theory of Operation}

A lumped element circuit model of the D-dot probe is shown in Figure 1. In the figure, $Z$ represents the impedance of the diagnostic cable attaching the probe to an oscilloscope or digitizer. $V_{S}$ is the signal registered on the oscilloscope in response to a drive voltage $V_{D}$ applied to the electrode. The probe is coupled o the charging electrode through a capacitance, $C_{c}$, and to the wall, nominally the ground electrode, 
through a capacitance, $C_{w}$. The $Q$ 's represent the surface charges needed to balance the applied voltages. $V_{S}$ is generated by the displacement current, $I$, from the movement of these charges flowing through the impedance. Note that the charges, voltages, and current are all time dependent while the capacitances and impedance are not. Symbols with definitions are listed in Appendix A.

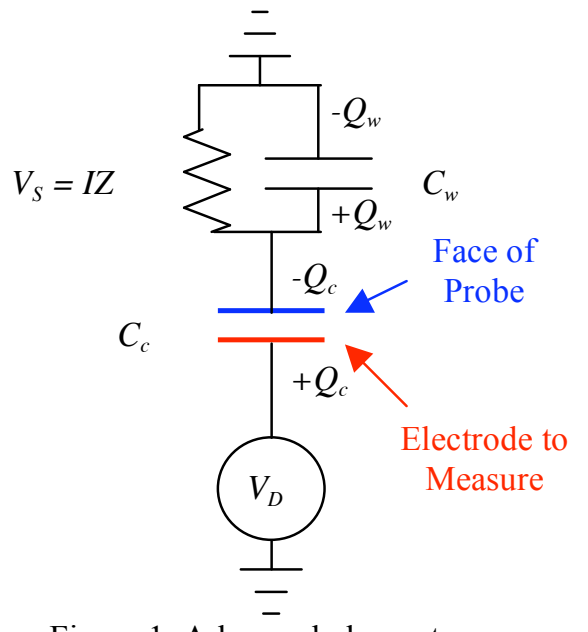

Applying Kirchoff's Voltage Law and the conservation of charge to the circuit model we find:

$$
\begin{gathered}
V_{S}=I Z=\frac{Q_{w}}{C_{w}}, \\
V_{D}=\frac{Q_{c}}{C_{c}}+V_{S}, \text { and } \\
I=\dot{Q}_{c}-\dot{Q}_{w}
\end{gathered}
$$

Substituting Equations (1) and (2) into Equation (3) and rearranging terms:

$$
\begin{gathered}
\dot{V}_{D}(t)=\frac{C_{c}+C_{w}}{C_{c}} \dot{V}_{S}(t)+\frac{1}{Z C_{c}} V_{S}(t), \text { or integrating, } \\
V_{D}(t)=\frac{C_{c}+C_{w}}{C_{c}} V_{S}(t)+\frac{1}{Z C_{c}} \int_{0}^{t} V_{S}\left(t^{\prime}\right) d t^{\prime}+V_{D}(0) .
\end{gathered}
$$

There is a subtle assumption that all free charges are induced by the applied voltage $V_{D}$. This assumption may not be true in the presence of a dielectric as will be discussed below in the Detailed Analysis Section. I have also assumed that $V_{D}$ for $\mathrm{t} \leq 0$ is constant so that $V_{S}(0)=0$. Equation (5) is used to convert the measured signal into the applied voltage. However, Equation (4) is useful in predicting the response of the probe to a known applied voltage during the design of the diagnostic.

\section{Electrical Design}

The mechanical design of the D-dot probe involves a number of issues. If the probe is installed through a vacuum interface, the vacuum properties of the probe materials as well as vacuum integrity of the interface need to be addressed. Structural compatibility must also be considered. For this report, only the electrical design issues are discussed. The electrical performance issues can be separated into signal magnitude and frequency response.

\section{Probe Response - Scope signal compared to drive voltage}

Many voltage pulses can be approximated as a linear ramp to a constant value. Consider Equation (4). If we assume that the rise/fall time is a linear ramp so that $\dot{V}_{D}$ is constant. Then

$$
\begin{gathered}
\dot{V}_{S}(t)+\frac{1}{Z\left(C_{c}+C_{w}\right)} V_{S}(t)=\frac{C_{c}}{C_{c}+C_{w}} \dot{V}_{D}, \text { or } \\
V_{S}=Z C_{c} \dot{V}_{D}\left(1-e^{-t / \tau}\right), \text { where } \tau=Z\left(C_{c}+C_{w}\right) .
\end{gathered}
$$


The derivation of Equation (7) is given in Appendix B. Thus the magnitude of the diagnostic signal is proportional to the coupling capacitance. The time response, $\tau$, or bandwidth, of the probe is determined by the sum of the capacitances, $C_{c}$ and $C_{w}$.

Another approach to the probe's response is to consider a sinusoidal drive voltage. If

$$
\begin{gathered}
V_{D}(t)=V_{D} \sin (\omega t), \text { and } \dot{V}_{D}(t)=\omega V_{D} \cos (\omega t), \text { then } \\
V_{S}(t)=\frac{Z C_{c} \omega V_{D}}{\omega^{2} \tau^{2}+1}\left[\omega \tau \sin (\omega \tau)+\cos (\omega \tau)-e^{-t / \tau}\right]
\end{gathered}
$$

The derivation of Equation (9) is given in Appendix B. For $t>>\tau$ so that the exponential term may be ignored:

$$
\frac{\left\langle V_{S}\right\rangle}{\left\langle V_{D}\right\rangle}=\frac{Z C_{c} \omega}{\left(\omega^{2} \tau^{2}+1\right)^{1 / 2}}=S_{21},
$$

where $S_{2 I}$ would be the signal response between the output and input ports of a network analyzer. Again, the magnitude of the diagnostic signal is proportional to the coupling capacitance while the frequency response is related to $\tau$.

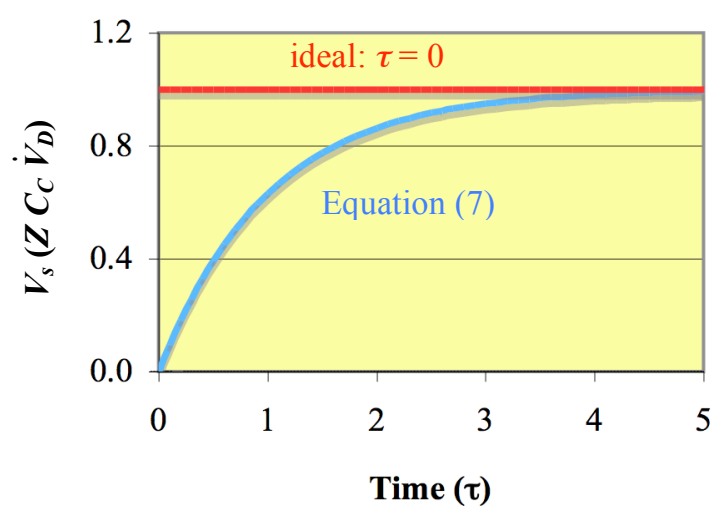

Figure 2. Probe response for ramped drive.

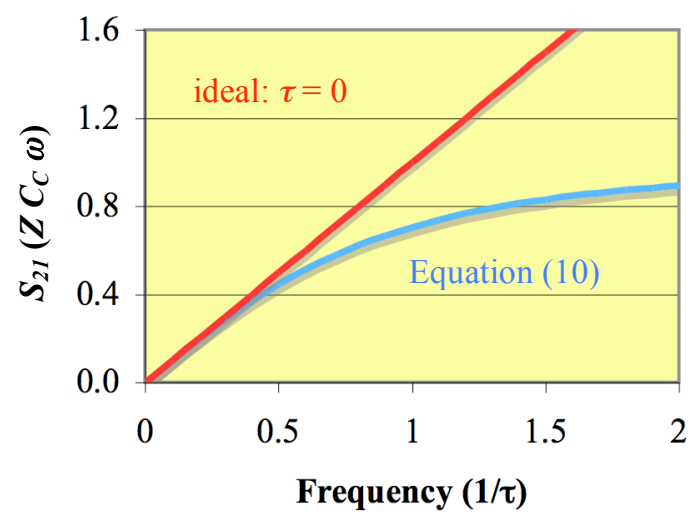

Figure 3. Probe response for a sinusoidal drive.

In Figures 2 and 3, response curves from Equations (7) and (10) are plotted to show the effect of a nonzero time constant. Both figures present the same information, simply emphasizing different aspects of the time response. Basically, to be good to $5 \%$, we need to measure the ramp for more than $3 \tau$, limit the frequency components of our signal to less than $1 / 3 \tau$, or post process the signal to account for the time constant. Note that post processing is limited. If the high frequency components are attenuated until their levels are comparable to the noise level, the information is lost. Consideration of the expected frequency components of the drive voltage provides an estimate of the sum of the capacitances: $\tau=Z\left(C_{c}+C_{w}\right)$.

High voltage diagnostics need to produce an output signal significantly smaller than the voltage being measured. Using Equation (7) and ignoring the exponential response,

$$
V_{S}=Z C_{c} \dot{V}_{D} \approx Z C_{c} \frac{V_{d}}{\Delta}, \text { or } C_{c} \approx \frac{\Delta}{Z} \frac{V_{S}}{V_{d}}, \text { where }
$$


$V_{d}$ is the flat top voltage and $\Delta$ is the ramp time to reach $V_{d}$. Equation (11) provides a quick estimate of the desired coupling capacitance.

\section{Practical Considerations}

Available "good" diagnostic cables will force the impedance to $50 \mathrm{ohms}$. A few other impedance cables exist, but high quality, low loss, matched cables and connectors are $50 \mathrm{ohms}$. Converters for fiber links are designed for $50 \mathrm{ohms}$. Fast digitizers for signal acquisition are also based on $50 \mathrm{ohms}$. It is important to eliminate any impedance mismatches in the path of the probe to the recording instrument as reflections can completely destroy the signal. Thus, $Z=50 \mathrm{ohms}$. Appendix C provides a sampling of $50 \mathrm{ohm}$, semi-rigid, copper jacked, coaxial cable.

The next practical consideration is that these probes are intended for use as a diagnostic for electrical breakdowns. Normally the electrode voltages will be on the order of tens of kilovolts or larger, i.e. $V_{s} / V_{d} \sim$ $10^{-4}$. The use of high frequency attenuators in the diagnostic line can be of value in this situation. Also, for very narrow electrode spacing, this requirement may not be true allowing more freedom in the design. Otherwise, from Equation (11), $C_{c}<<\mathrm{nF}$. As will be discussed below, the small physical dimensions of the probe can usually lead to a very small $C_{c}$ ensuring a reasonable magnitude for $V_{s}$. However, a quick estimate using Equation (11) should be performed early in the design.

Electrical breakdowns generate substantial high frequency electromagnetic pulses (EMP) that can couple to other electronics including diagnostics. The quantity of energy discharged and the peak power in the breakdown are an excellent metrics for the difficulty and required effort to shield equipment from the EMP. In addition to shielding, differential probes can be used to mitigate the EMP problem. Stygar ${ }^{2}$ describes such a probe design for an unusually harsh environment. The logic behind the differential probes is that two identical probes are placed side by side with one probe shielded such that it should receive a null signal. Subtracting the signals of the two probes will remove any common modes or noise signals.

Size is a critical feature for the specific application of monitoring the electrical breakdown. The probe must be positioned as near as possible to where the electrical breakdown is anticipated. This will minimize the normally negligible stray capacitances and inductances that seriously distort the desired signal due to the short time scale of the initiating event. However, the probe itself should not distort the electric field where the event occurs. The coaxial probe geometry described below behaves like a quadrupole in the far field, so that the distortion of the field falls off like $\mathrm{r}^{-3}$. As a quick estimate use

$$
(\mathrm{a} / \mathrm{r})^{3}=\text { desired accuracy, }
$$

where $a$ is the radius of the probe's dielectric, $r$ is the distance to the event of interest. For example, if the desired accuracy is $1 \%$ and the probe distance to the event is $1 \mathrm{~cm}$, the probe's radius should then be less than $(0.01)^{1 / 3}(1 \mathrm{~cm})$ or $2.15 \mathrm{~mm}$. 
There are two practical limitations in regards to the physical smallness of the probe. The first is that size determines the difficulty of fabrication, which is equivalent to cost. An inexpensive probe can be constructed by cutting off the end of a coaxial cable. Unfortunately, that technique allows little control of the geometry resulting in smeared, deformed, and/or sharp edges. Fabrication tolerances can be relaxed if the final dimensions are measured after fabrication and/or in situ calibrations performed. Issues related to field enhancements due to gaps, edges, etc., can be reduced by judicious location of the probe with respect to electrodes and insulators.

A second limitation associated with very small dimensions is the frequency dependent, signal attenuation due to ohmic wall losses. Post processing of the probe signal can correct for some of this attenuation, as will be demonstrated below. However, those signal components that are attenuated until they are comparable to the noise level cannot be accurately reconstructed. The amount of attenuation may be lessened if the signal is transferred to larger diagnostic cables and/or converting to fiber optic cables. A matched impedance section is required for the transition.

A major practical limitation is recording and calibrating the output of the probe. The present limits of commercial digitizing oscilloscopes and network analyzers are about $12 \mathrm{GHz}$ and $20 \mathrm{GHz}$, respectively. Calibration requires a signal source with known frequency components equal or greater than the range of interest. Even with a reliable signal source, an appropriate fixture to couple the source to the probe remains a challenge. In the Airline Calibration section the difficulties are made obvious. The calibration of a probe, with an expected bandwidth of greater than a $100 \mathrm{GHz}$, is limited by the $20 \mathrm{GHz}$ signal generator of the network analyzer and further limited to less than $4 \mathrm{GHz}$ by the calibration fixture (airline). An accurate calibration to $20 \mathrm{GHz}$ would require resources comparable or greater than those required for fabrication of the diagnostic.

\section{Calculating Capacitances}

There are various techniques for calculating capacitances based on energy or surface charge. Appendix D provides some formulae for estimating capacitances, but the final design values should be determined through an electrostatic solver. For the coupling capacitance, it is important to include the coupling from both the outer radius as well as the exposed end of the inner conductor. The "wall" capacitance does not include the coaxial cable capacitance. The cable capacitance is included in the impedance, $Z . C_{w}$ is primarily the coupling from the exposed end of the inner conductor to the wall. As will be discussed in the example design, a total capacitance including both $C_{w}$ and the cable capacitance is determined by electrostatic simulation. The portion due to the cable is then calculated and subtracted to give $C_{w}$. Note that the coupling capacitance is related to the total geometry, especially the spacing between the electrodes while the wall capacitance depends primarily on the dimensions of the probe. 


\section{Tolerances}

In general the response of the D-dot probe does not require extremely tight tolerances if an in situ calibration can be performed. A modest 2 mil requirement with respect to the probe face relative to the electrode can produce $\sim 25 \%$ variation from the design performance/calibration due to shielding and enhancements. This variation should be sufficient to produce a probe within the dynamic range of the recording equipment. Since the coupling capacitance is also determined by the position of the probe with respect to the surrounding electrodes, an in situ calibration should be considered necessary. This is particularly true for inherently 3-dimensional geometries where computational resolution could be an issue. Calculating the design parameters for a 2-dimensional approximation to the real geometry then performing an in situ calibration can be the most efficient approach. Electrostatic modeling of sample positional errors is provided in the next section.

If an in situ calibration cannot be performed, tolerances will be an issue. In this case, several prototype probes could be constructed and calibrated in a test fixture to determine the reproducibility.

However, the primary tolerance issues involve gaps/spaces that can trap air and/or enhance the local electric field. Probes that are located under the insulator can act as virtual leaks during the evacuation of the test chamber. When the high voltage is applied, the trapped gases can become ionized producing plasma that could short the probe or produce an electrical breakdown in the system. Best practices for avoiding trapped gasses need to be employed.

\section{Design Example}

A D-dot probe for a high voltage, vacuum insulator test chamber is described in this section. Cost and schedule considerations were the principle factors for using a commercial cable. However, the probe must generate a useable signal without impacting the performance of the test chamber. A few preliminary design questions need to be considered:

1. What phenomenon is being measured? This question addresses the sensitivity of the probe and location. For this example, the time of the flashover of the insulator after exposure to a pulse of UV radiation was required to nanosecond accuracy. This basic requirement is not difficult and might be accomplished with an appropriate light detecting diode system. However, for nanosecond accuracy the probe should be located in the electrode gap. A desired goal was to detect the electrical fields due to the movement of electrons across the insulator surface. This is challenging and requires a fast, very small D-dot that can be located near the point of flashover on 
the insulator surface. To avoid interception of electrons of electrons during the flashover, the probe should be under the insulator rather than in the vacuum gap.

2. Will the probe be installed in the anode or cathode? With a cut commercial cable, edges will not be beveled and localized high (enhanced) electric fields will result. Even with a carefully fabricated probe, some field enhancement will occur. This is not an issue on the anode, but could lead to explosive plasma formation and breakdown from the cathode surface. Locating the probe under the insulator mitigates the problem.

3. Will the probe pass through a vacuum interface? What are the vacuum requirements? High vacuum will require attention to the dielectric used in the probe and virtual leaks. Commercial high vacuum feed through connections are available, but their physical dimensions can be expected to be incompatible with the mechanical design. In this case, the probe would need to be entirely fabricated. This provides a superior diagnostic compared to a simple coaxial cable, but at much greater cost.

4. How much energy is stored in the system and could be coupled to the probe? The stored energy determines if protective measures need to be engineered into the diagnostic circuit to provide personnel and/or equipment protection. The stored energy also indicates the level of EMP mitigation required.

Fortunately, the vacuum requirements for the test chamber are modest and the probes could be embedded in the anode without breaking a vacuum interface. The maximum stored energy in the system was less than $300 \mathrm{~mJ}$ and only a fraction of that energy could be expected to couple to the probe. Thus, adapting a commercial cable for use as a $\mathrm{D}$-dot probe is reasonable.

\section{Initial Estimates}

Consider a probe to be used in an insulator test where the insulator has a dielectric constant of 2.3, the parallel electrodes are spaced $1 \mathrm{~cm}$ apart, the maximum potential between the electrodes will be $100 \mathrm{kV}$, and the flashover is expected to occur on a time scale of 500 picoseconds. Assuming a maximum desired signal on the diagnostic line of 10 volts, Equation (11) estimates a desired coupling capacitance of $1 \mathrm{fF}$. Equation (D-3) can then be used to estimate an inner conductor radius of $0.23 \mathrm{~mm}$ or diameter of $0.46 \mathrm{~mm}$. RG405/U semi-rigid coaxial cable, referred to as " 085 cujack" due to its outer diameter in mils, is the nearest commercial cable listed in Appendix $\mathrm{C}$ to these parameters.

The next check considers the distortion of the electric field on the insulator. Assume the closest distance from the center of the probe to the insulator surface is $9 \mathrm{~mm}$ and the radius of the probe is $0.838 \mathrm{~mm}$ (RG405/U), the distortion of the field is approximately $(0.838 / 9)^{1 / 3}$ or about $0.08 \%$. 


\section{Electrostatic Modeling: Capacitances}

The geometry for the electrostatic simulation is shown in Figure 4. The radius of the inner conductor is $0.255 \mathrm{~mm}$, the radius of the outer conductor is $0.838 \mathrm{~mm}$, and the cable's dielectric constant was 2.1. The probe is situated under the insulator (dielectric constant of 2.3) with a cathode/anode gap of $1 \mathrm{~cm}$. As mentioned above, there are different ways of determining the capacitive coupling between the different surfaces. I choose to use the induced surface charge:

$$
C=\frac{Q}{V}=\frac{\varepsilon \oiint E_{\perp} d A}{V} .
$$

For the coupling capacitance, the probe tip and anode were at ground while the cathode was at $100 \mathrm{kV}$. The electrostatic solver calculated $Q, 0.1413 \mathrm{nC}$, yielding a capacitance, $C_{C}=1.4 \mathrm{fF}$.

If the probe tip and cathode are held at the same potential and the anode is set at a potential difference, $\mathrm{V}$, relative to the probe, a total capacitance, $C$, comprised of both the wall, $C_{w}$, and cable, $C_{\text {coax }}$, capacitances is determined:

$$
\begin{gathered}
C=C_{\text {coax }}+C_{w}=503.3 \mathrm{fF}, \text { where } \\
C_{\text {coax }}=\frac{2 \pi \varepsilon \Delta z}{\ln \left(r_{o} / r_{i}\right)}=477.6 \mathrm{fF}
\end{gathered}
$$

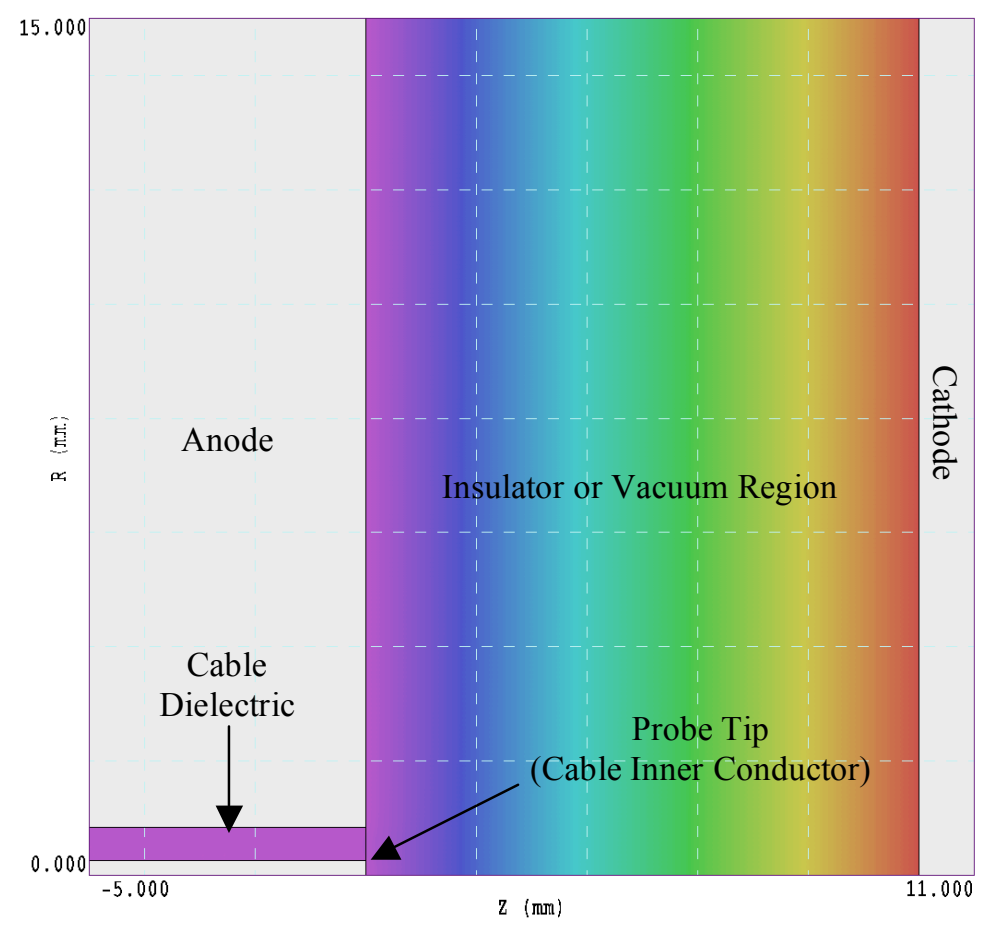

Figure 4. Electrostatic simulation geometry (anode and probe are at equal potentials) is shown with equipotential contours.

for the $\Delta z(5 \mathrm{~mm})$ coaxial segment used in the simulation. Thus, $C_{w}$ is $25.7 \mathrm{fF}$. The values shown in Table 2 of Appendix $\mathrm{C}$ were calculated by this technique.

The probe tip was cut flat with respect to the anode wall in the simulation. This leads to significant field enhancement on the edges as shown in the left simulation of Figure 5. The peak field was lowered by more than a factor of two, from $505 \mathrm{kV} / \mathrm{cm}$ to $223 \mathrm{kV} / \mathrm{cm}$, in the right simulation by beveling the edges with a radius of curvature equal to half the inner radius of the probe. The capacitances changed by $<0.5 \%$.

Beveling the edges is a major mechanical effort and cost. The dimensions are small and the machining would need to be accomplished with out the dielectric. Once machined, the inner conductor would have to be positioned and an appropriate epoxy/potting compound added. Surfaces would then be cleaned and a connection point for the diagnostic cabling added. While all of this is possible, techniques for mitigating the effects of field enhancements should be considered as a viable alternative. 

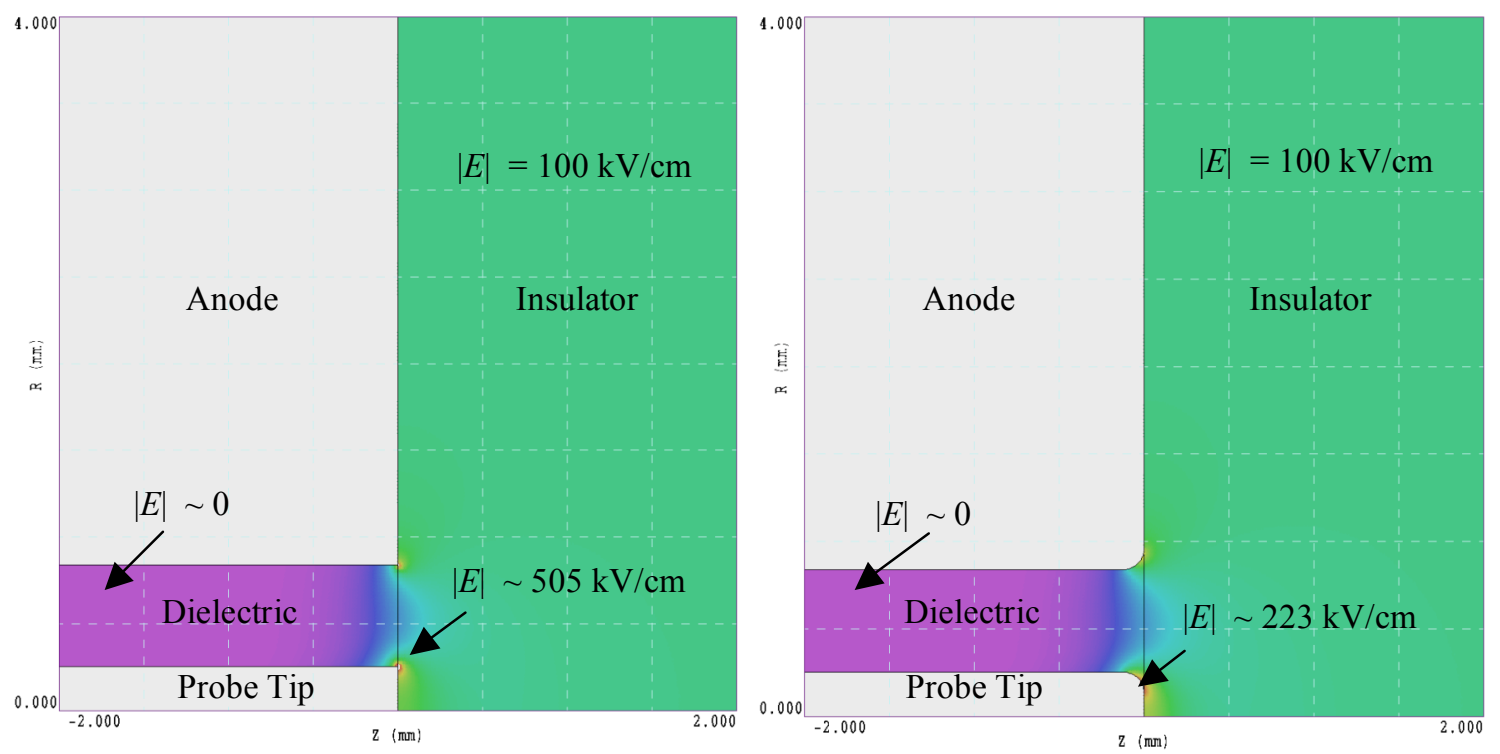

Figure 5. Close up views of probe tip geometries, flat end on left and beveled (radius) tip on right. Color coding indicates electric field magnitude.

\section{Electrostatic Modeling: Errors}

Tolerances can be easily established from electrostatic modeling. Figure 6 illustrates the effects of misalignment of the probe tip with respect to the surface of the electrode. For the RG405/U dimensions, recessing the probe tip by $0.05 \mathrm{~mm}$ ( 2 mils) decreased the coupling to the cathode by $20 \%$ and the wall capacitance by $14 \%$. Note that there was a vacuum gap between the end of the probe and the insulator for this simulation. If the probe extended above the anode by 0.05 mils, coupling to the cathode increased $26 \%$ and the wall capacitance increased $14 \%$. For this simulation, a vacuum gap existed between the insulator and the anode.
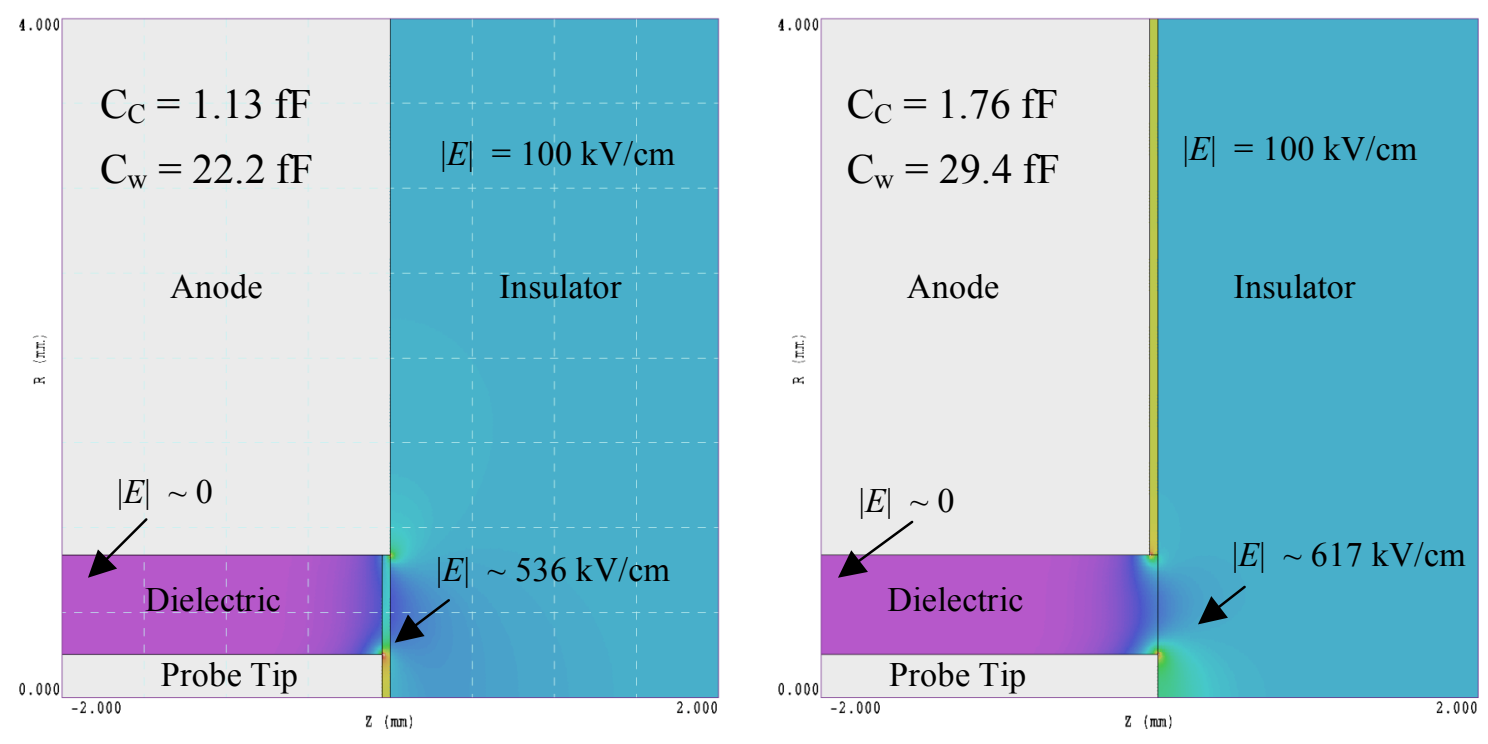

Figure 6. Close up views of simulated alignment errors, probe recessed 2 mils on left and extended on right. 
The effect of an offset coaxial inner conductor (probe tip) was studied with a 3-D electrostatic code. The simulations results for a 4-mil off centered inner conductor are shown in Figure 7. Fortunately, the probe calibration parameters are much less sensitive to errors for the more difficult centering of the probe tip than for the positioning with respect to the electrode surface.
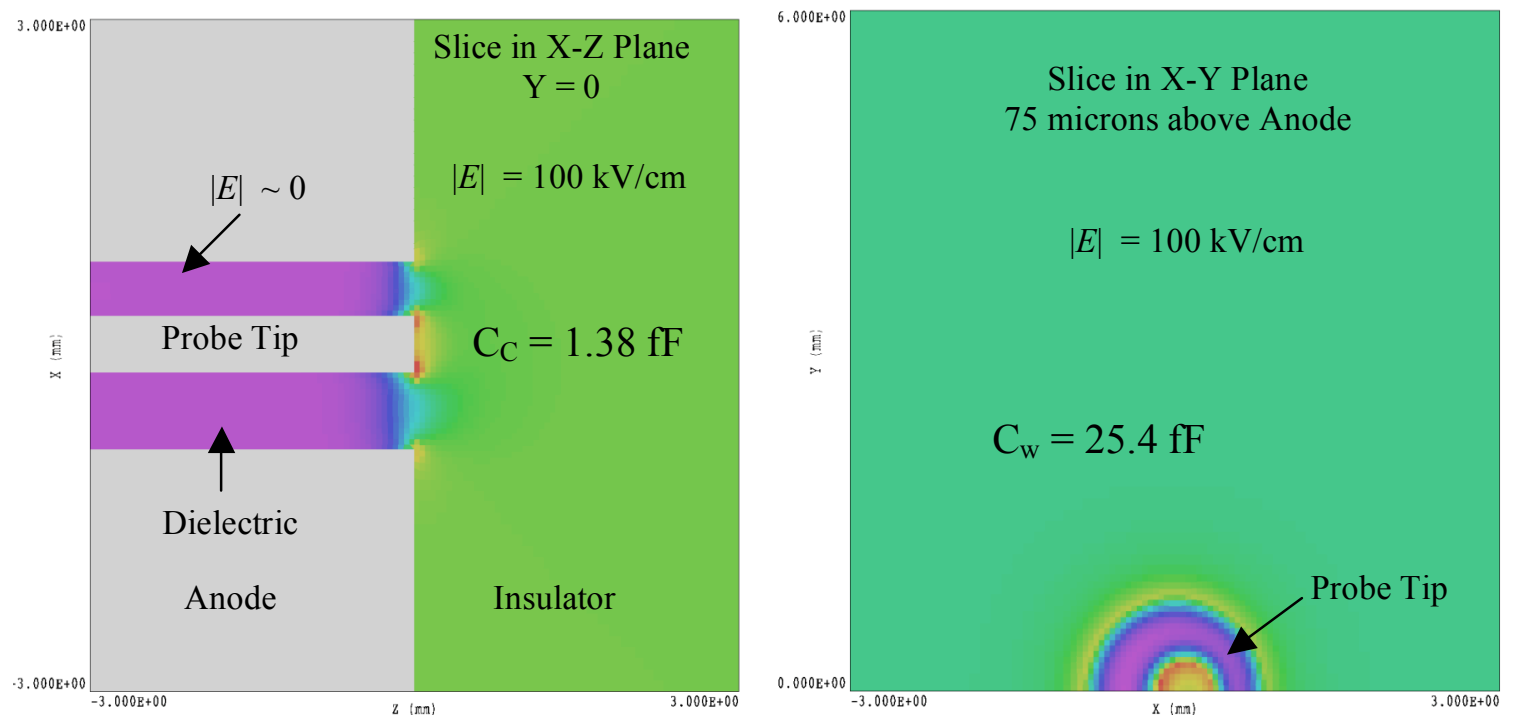

Figure 7. Close up views of a simulated 4-mil coaxial alignment error, side view on left and top view on right. Color coding indicates electric field magnitude.

\section{Airline Calibration}

A preliminary calibration was performed on an airline. An airline in this sense is simply a coaxial cable with varying diameters, but constant impedance. The central part of the airline has a relatively large diameter compared to standard cable to allow the insertion and testing of various probes. The diameter of the airline tapers at the ends to accept standard sized connectors. A photograph of the airline and test probe is shown in Figure 8. In operation, a signal, normally sinusoidal, is applied to one end of the airline, transits in the fundamental TEM mode, and exits at the other end with no reflections. Single mode propagation in the TEM mode is possible from DC to frequencies such that the wavelength is approximately equal to the average circumference of the airline. At higher frequencies there will be the possibility of mode conversion and multiple modes transiting simultaneously. The airline used had dimensions that allowed single mode propagation to about $1 \mathrm{GHz}$.

\section{Calibration Data versus Expected Response}

An Agilent Technologies E8362B Network Analyzer was used to drive the airline and measure the power coupled to the D-dot probe referred to as a S21 measurement indicating that it is a ratio of the power measured at an output port (Port 2) to the input power (Port 1). The D-dot probe included 30 inches of 
coaxial cable as shown in Figure 8. The cable attenuates the probe signal and needed to be included in the response. The attenuation is often given as a frequency dependent term, $\alpha$, for a specific frequency in units of $\mathrm{dB}$ per distance:

$$
\alpha \Delta z=-10 \log \left(\frac{P}{P_{0}}\right)=-10 \log \left(\frac{V}{V_{0}}\right)^{2}=-20 \log \left(\frac{V}{V_{0}}\right)=\beta \sqrt{\omega} \Delta z .
$$

For the power levels and frequencies of interest, $\alpha$ is proportional to the square root of the frequency. In Equation (13) the frequency dependency is made explicit leaving a constant $\beta$ that can be measured or calculated. Attenuations for several semi-rigid coaxial cables are listed in Appendix C. Equation (10) then needs to be modified:

$$
S_{21}=\frac{\left\langle V_{S}\right\rangle}{\left\langle V_{D}\right\rangle}=\frac{Z C_{c} \omega}{\left(\omega^{2} \tau^{2}+1\right)^{1 / 2}} 10^{-\beta \sqrt{\omega} \Delta z / 20}
$$

The coaxial geometry of the airline is different than for parallel plates so the coupling capacitance must be recalculated. The problem is three-dimensional with a significant variation in scaling. A very accurate estimate is to use the area factor calculated for the probe for the parallel plate geometry and the surface field on the outer wall for the coaxial geometry. This is simply Equation (D-5) and the expected capacitance is $C_{c}=0.11 \mathrm{fF}$. The wall capacitance remains the same for both geometries, $C_{w}=13.4 \mathrm{fF}$.

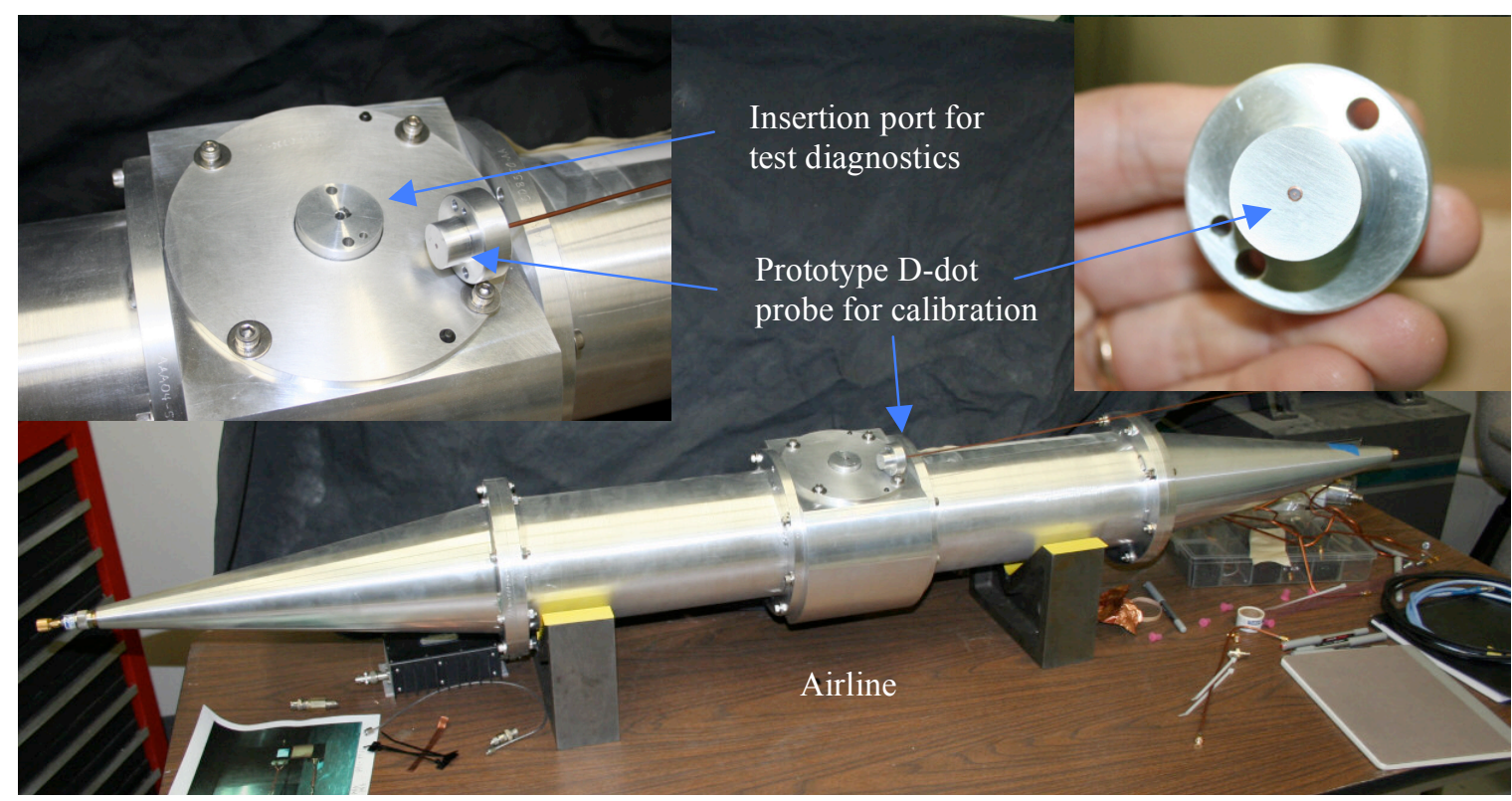

Figure 8. Photograph of calibration airline. Upper left insert is close up of the insertion port (blank flange in place). Upper right insert is close up of the D-dot probe. When inserted, the D-dot and probe face are flush with the inner wall of the outer conductor.

\section{Comments on Airline Calibration}

The airline was marginally suitable for the calibration. As can be seen in Figure 9, at frequencies above $1 \mathrm{GHz}$ mode conversion significantly affected the output and above $4 \mathrm{GHz}$ the airline was unusable. Also, 
the weak coupling below $1 \mathrm{GHz}$ was at the lower edge of the network analyzer operational range. With a $\tau$ of less than $0.7 \mathrm{ps}$, at $4 \mathrm{GHz}(\omega \tau)^{2}<3 \times 10^{-4}<<1$. Thus, Equation (14) reduces to:

$$
S_{21} \approx Z C_{c} \omega 10^{-\beta \sqrt{\omega} \Delta z / 20}
$$

The calibration did not provide any information on $C_{w}$ other than an upper bound approximately an order of magnitude larger than the calculated value. The fit to the measured data is sensitive to the value of $C_{c}$, and the calculated coupling capacitance is accurate to better than a few percent. This capacitance will be different for the parallel plate geometry, but the technique for calculating capacitance is validated. The manufacturer's published data for cable attenuation also was shown to be accurate.

For a satisfactory calibration, the radial dimensions of the airline needed to be reduced by a factor of 10 . For that case, the two capacitances would be approximately equal for a $\tau$ of $1.5 \mathrm{ps}$, the airline could operate in a single mode to $10 \mathrm{GHz}$, and the response would be about $20 \mathrm{~dB}$ greater. Both capacitances and the cable attenuation could be determined accurately

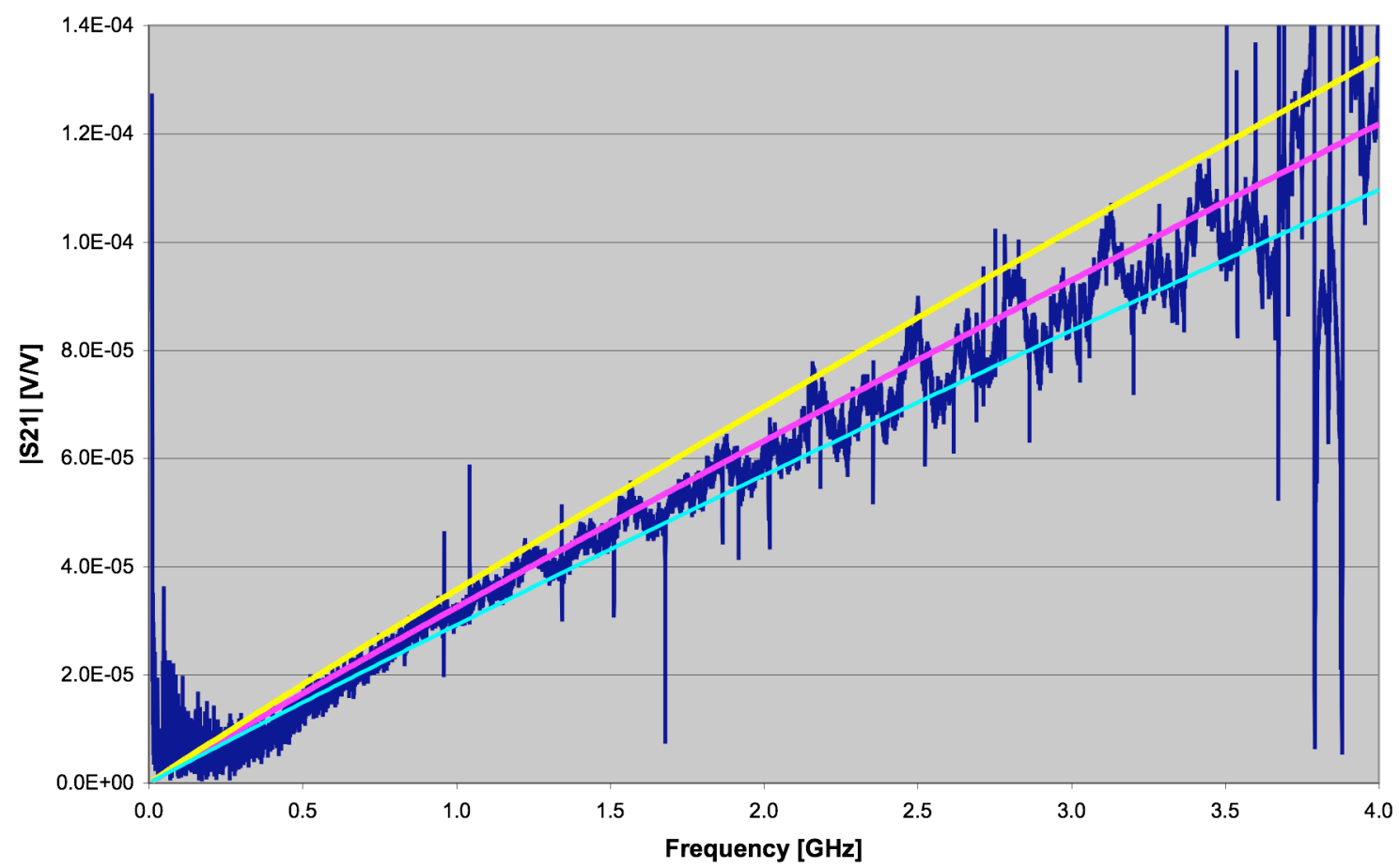

Figure 9. $\mathrm{S}_{21}$ measurement (dark blue) for the D-dot probe on the airline. The pink curve is the expected probe response, the yellow (turquoise) curve is the response for a $C_{c} 10 \%$ higher (lower) than calculated.

\section{Parallel Electrode Testing}

The parallel electrode testing was the application of the D-dot probes to the insulator test stand. A photograph of the anode with 5 probes is shown in Figure 10. A photograph of the inside of the test chamber with a HDPE insulator positioned between the electrodes is shown in Figure 11. The D-dot probes included 30 inches of the RG405/U cable as in the case of the probe tested on the airline. The length of the 


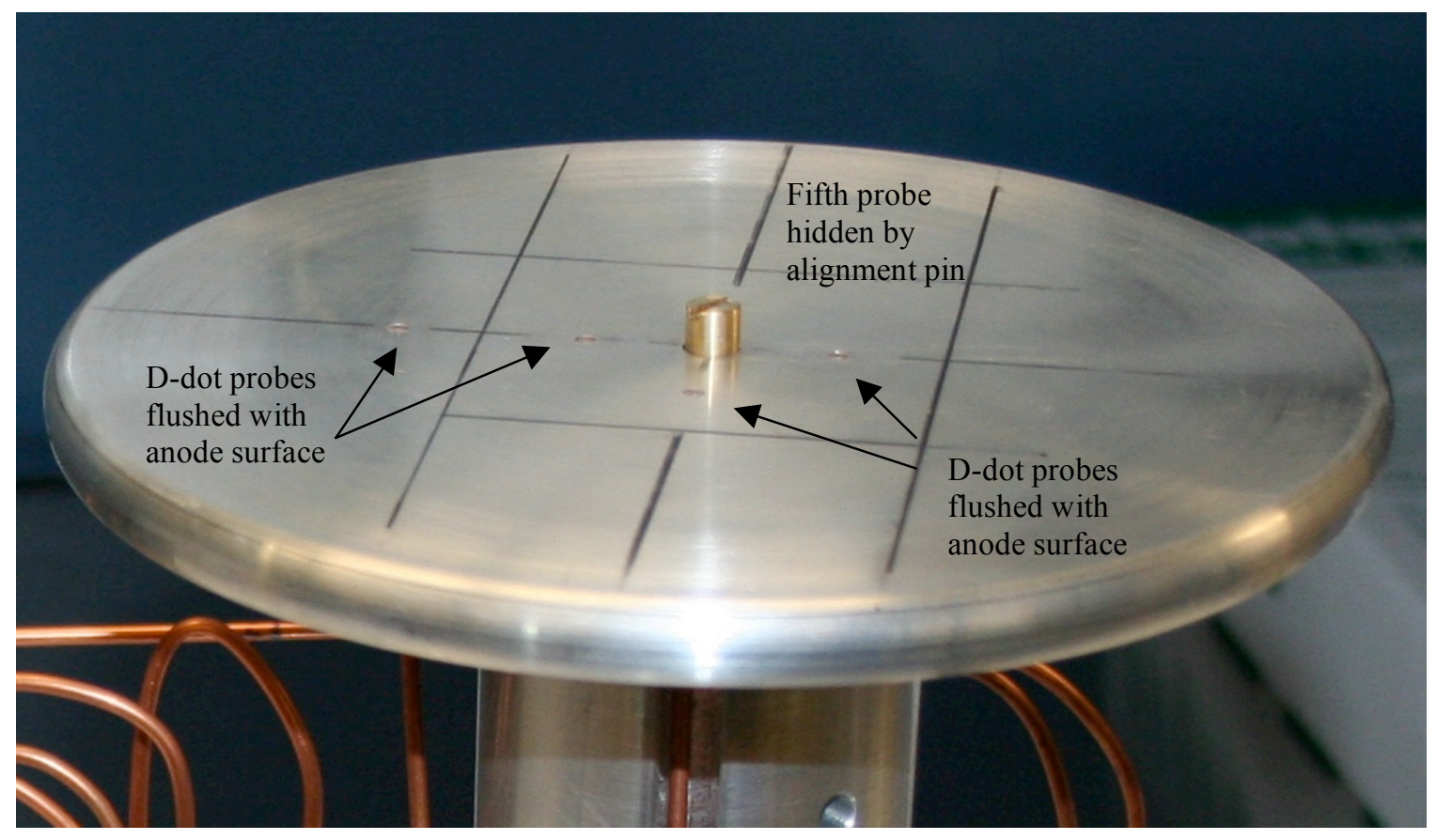

Figure 10. Photograph of the anode with four of the five D-dot probes visible. The black lines are spaced at $1 \mathrm{inch}$. The central square represents the maximum footprint of the tested insulators.

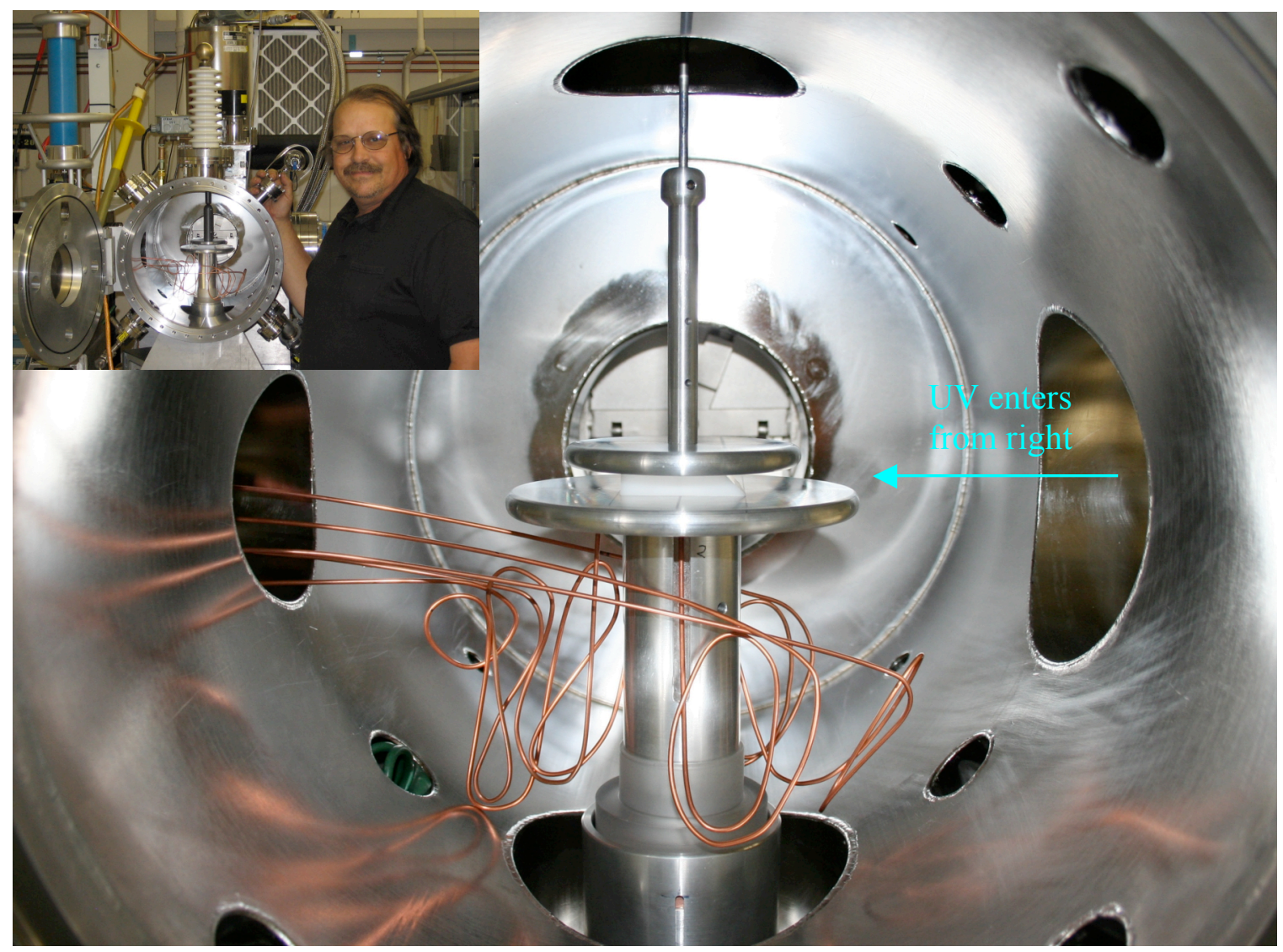

Figure 11. Photograph of the test chamber with a HDPE insulator positioned between the electrodes. The inset at the upper left illustrates the overall size of the test chamber. 
cables was chosen to allow flexibility in the installation of the anode and connection of the cables to a feed through plate in one of the ports of the chamber. The insulators tested were approximately square in cross section. The probes were located so that four were located under the insulator near the centers of the sides and one was located $\sim 1 \mathrm{~cm}$ away from the insulator. An Excimer laser pulse provided UV illumination of a square $\mathrm{cm}$ area on one side/face of the insulator to initiate flashover. Figure 12 is a schematic of the locations of the probes with respect to the test insulator and UV illumination. Note the insulator angles shown in Figure 12. When the insulator is positioned for testing at +45 degrees, the center of the probe is only $2.7 \mathrm{~mm}$ from the anode triple junction.
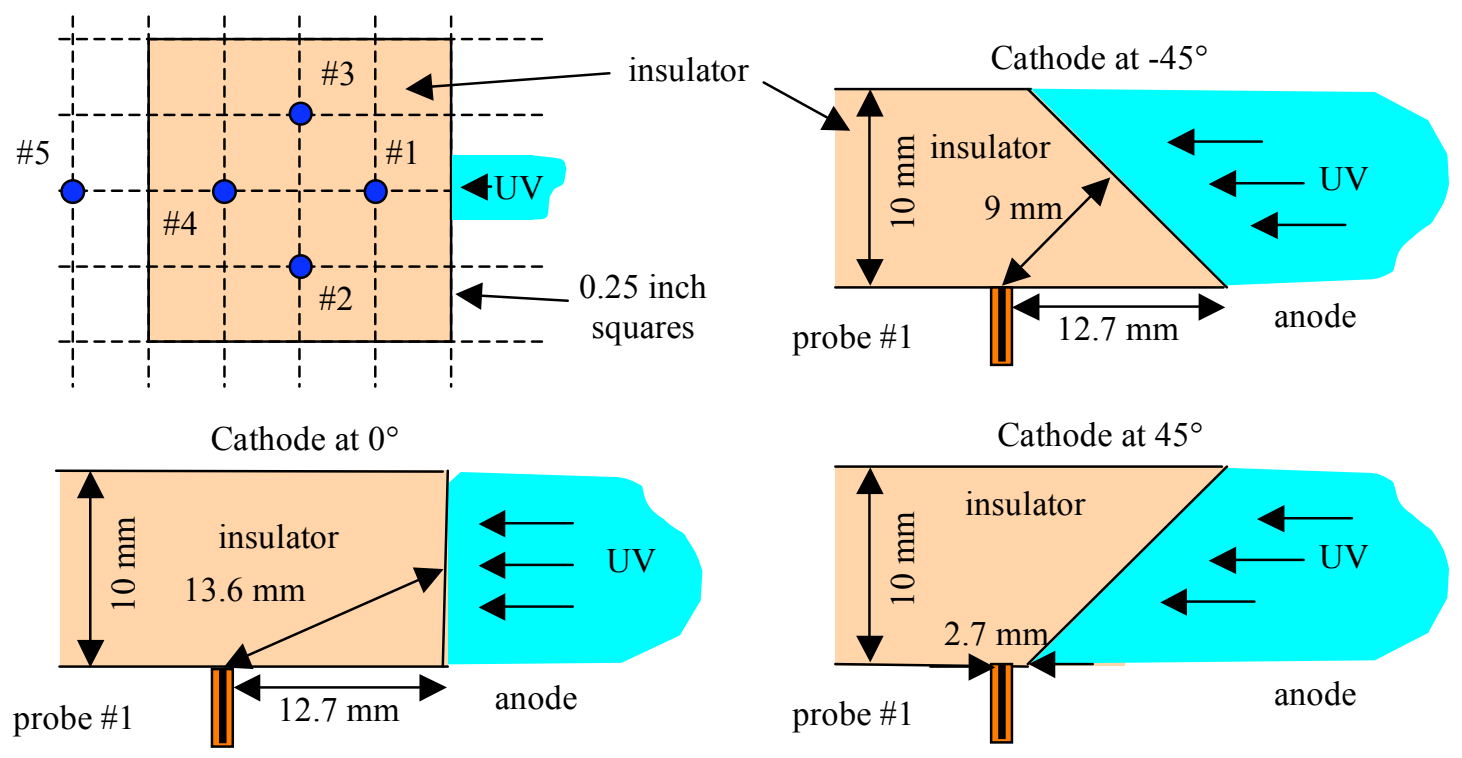

Figure 12. Schematic showing the D-dot probe positions with respect to the insulator and UV illumination.

\section{Raw Data}

A Tektronix TDS6124C $12 \mathrm{GHz}$ digitizing oscilloscope was used for acquiring the probe signals. The electrodes would be charged to a specified voltage and then a $1-\mathrm{cm}^{2}$ section of the insulator illuminated with UV. Normally the intensity of the laser pulse measured by a diode would be recorded on one channel of the oscilloscope and either 3 (6 GHz, 20 Giga samples/s) or 1 (12 GHz, 40 Giga samples/s) probe signal recorded on the remaining channels. Figure 13 shows the three probe traces of a single shot overlaid with a scaled laser pulse. The insulator for this test was Macor at $+45^{\circ}$. Total energy in the laser pulse was $66 \mathrm{~mJ}$ and the electrodes were charged to $45 \mathrm{kV}$. Cable and time of flight corrections have been applied so that the relative time is represented correctly between the laser and D-dot signals. The EMP from the flashover fed into the photo diode measuring the laser pulse and distorted the diode signal. All of the D-dot probes were nominally constructed the same. However, the coupling and wall capacitances depend on the local geometry and dielectric constants. For example, Probe $\# 5$ is not under the insulator, so its signal is approximately $1 / \varepsilon_{r}$ smaller than the other probes. Probe \#1 is located under the section of insulator illuminated by the UV. 

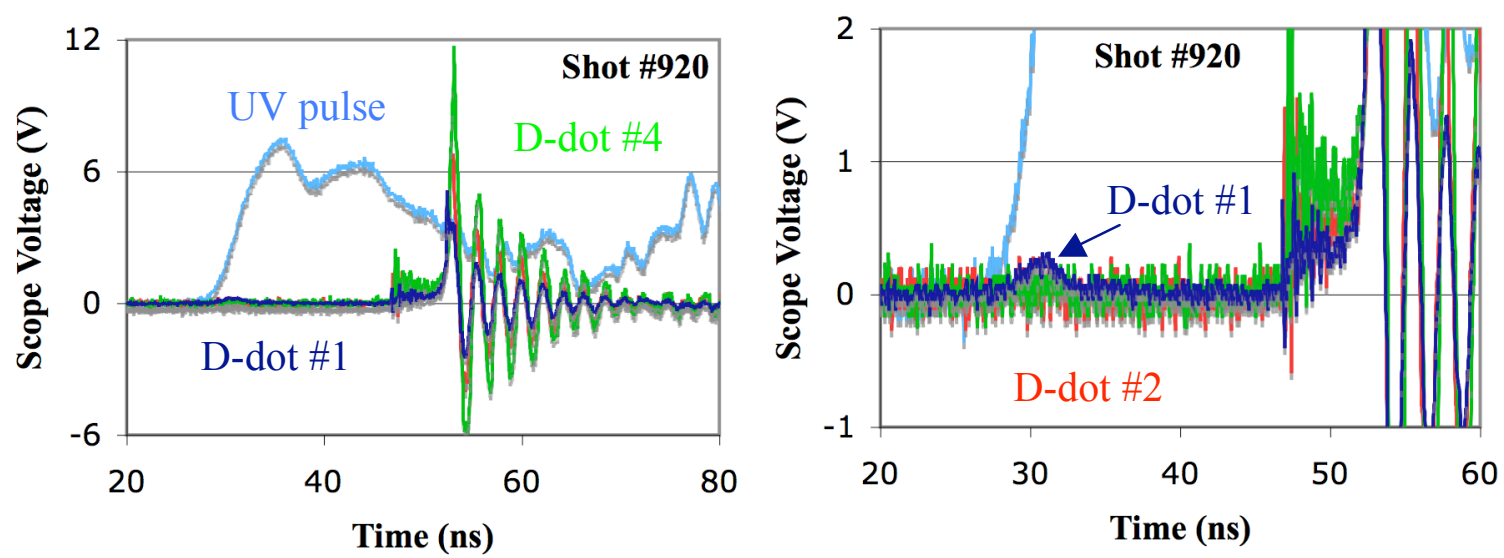

Figure 13. Raw D-dot probe signals overlaid with the UV laser pulse for two vertical scales.

The raw signals provide all the timing information needed for the insulator tests. Within $2 \mathrm{~ns}$ of the start of the laser UV pulse a small current can be detected on D-dot Probe \#1 as electrons are ejected from the insulator surface. The probe signals also indicate an interval of about 2 to $5 \mathrm{~ns}$ of activity before the flashover. Once the flashover occurs, the electric fields between the electrodes oscillate based on the LRC parameters of the overall chamber. An example of timing data acquired from the D-dot probes for experiments performed on Macor with a +30 degree angle is given in Figure 14. The charge voltage is the initial potential between the electrodes. The vertical axis shows the length of time from the beginning of the UV laser pulse until the collapse of the electrode potential as determined by the start of the large peak on the D-dot probe signals. The decreasing time with charge voltage indicates less UV energy is necessary to initiate flashover for high electrode potentials.

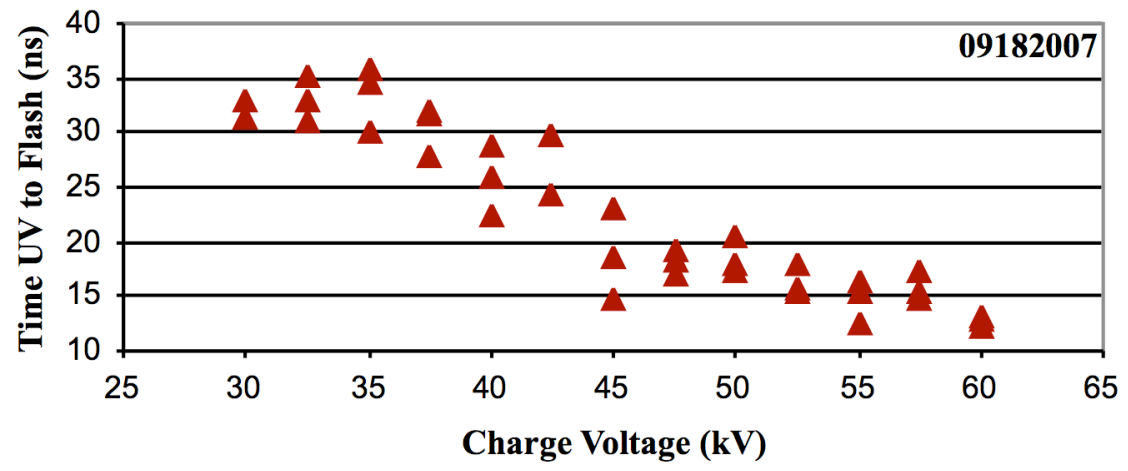

Figure 14. Example of timing data acquired from the D-dot probes.

\section{Correcting For Attenuation and Converting to Diode Voltage}

Correction for the frequency dependent cable attenuation is necessary before attempting a quantitative analysis of the D-dot signals. The probe signals are first corrected for initial dc offsets and Fourier Transformed into the frequency domain. The individual frequency components can then be corrected as was done for the airline data. The signal is then transformed back to the time domain. Figure 15 shows the results of the attenuation correction. The insulator for this test was Macor at $+30^{\circ}$. Total energy in the laser pulse was $35 \mathrm{~mJ}$ and the electrodes were charged to $52.5 \mathrm{kV}$. Only the laser diode and D-dot Probe \#1 
signals were recorded at 40 giga samples per second. The correction is subtle increasing the amplitude of the sharpest peaks. As will be discussed below, this effect is primarily important for determining the rate of charge movement.

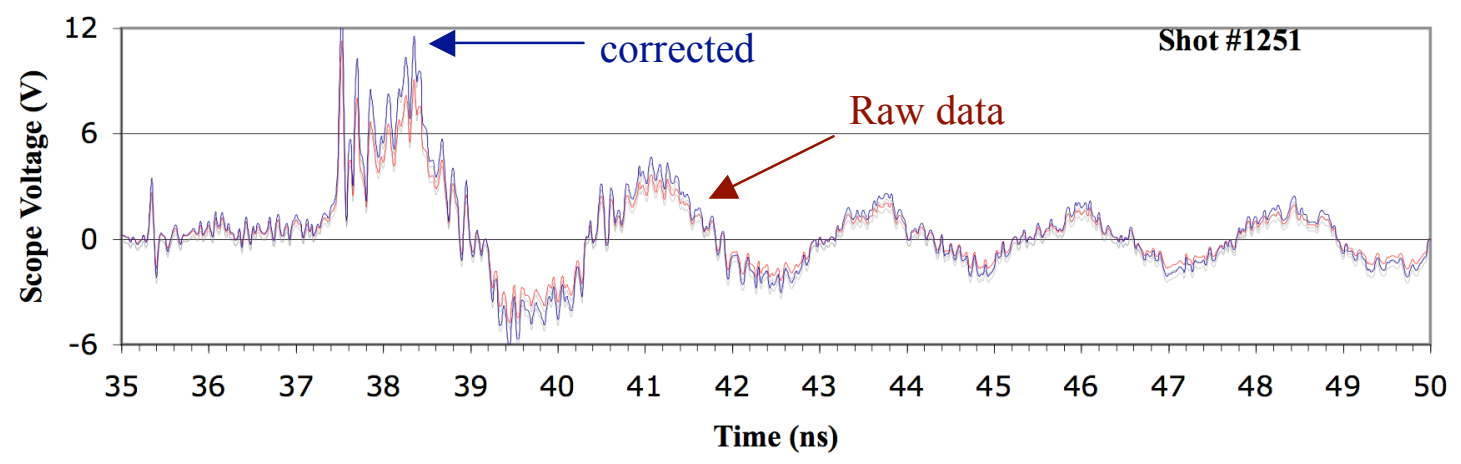

Figure 15. A comparison of the probe signal before and after correcting for cable attenuation.

Equation (5) can be used to convert the probe signal into voltage across the electrodes. For this particular application, the initial and final voltages are known, so what is determined is the time variation of the diode voltage during the flashover event. Note that the data can be used to verify the coupling constant. Rewriting Equation (5) and integrating to a time $t$ where $V_{S}(t)$ and $V_{D}(\mathrm{t})=0$, i.e. the system has fully discharged:

$$
C_{c}=-\frac{\int_{0}^{t} V_{S}\left(t^{\prime}\right) d t^{\prime}}{Z V_{D}(0)}
$$

Converting the corrected signal in Figure 15 with Equation (5) yields the diode voltage shown in Figure 16.

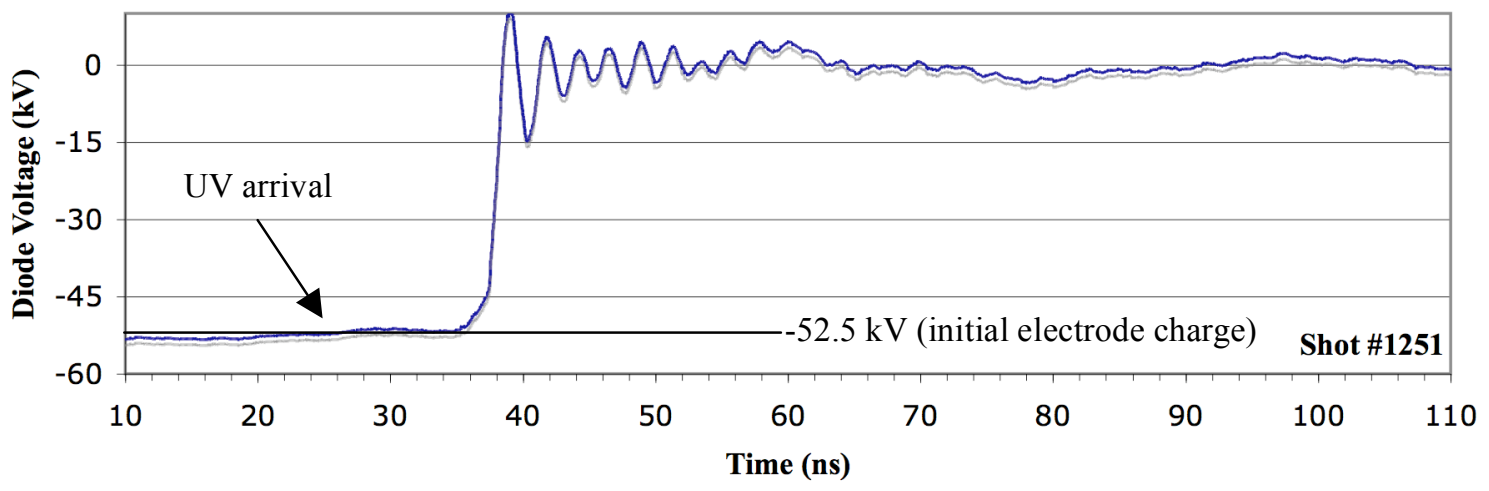

Figure 16. D-dot probe signal converted to the diode voltage during the insulator flashover.

\section{Detailed Data Analysis}

D-dot probes are often considered to be voltage monitors. In reality the probes are sensors of the local electric field magnitude. By combining the magnitude of the field with a predetermined field pattern allows the voltage to be determined. The coupling capacitance is simply reducing the field pattern to a scalar quantity that relates an applied voltage to the magnitude of the field, or induced charge, on the probe. The application of D-dot probes to the study of insulator flashover is difficult since the field pattern is not known a priori. The only quantity that is measured is the charge induced on the probe. 
The pertinent question is what can be determined about an insulator flashover using the D-dot probes that is useful to studying the flashover. There were three important pieces of information that could be gleamed from the probes during the insulator testing. Measuring the arrival signals (electric fields) at an array of probes permits the location of the source to be determined by triangulation. If the source location is known, the induced charge at the probe can be used to determine the magnitude of that source charge. The time dependent pattern of surface charging as a function of insulator angle or material should be related to the flashover mechanism. Simulations based on proposed mechanisms would need to be performed and compared to the data. Although it may not be possible to definitively prove a specific mechanism, those flashover mechanisms that produce results incompatible with experiment can be eliminated.

Below the effectiveness of the D-dot probes for studying insulator flashover is described.

\section{Flashover Site}

Multiple probes were used in the test stand anode to determine if the location of the flashover could be determined. The small size of the insulator was an issue. The greatest distance between probes was $2.54 \mathrm{~cm}$ for probes under opposite insulator faces. The maximum time interval between signal arrival between these probes would be $\sim 210$ ps:

$$
\Delta \mathrm{z} \sqrt{\varepsilon_{r}} / c=2.54 \mathrm{~cm} \frac{\sqrt{6}}{3 \times 10^{10} \mathrm{~cm} / \mathrm{s}}=210 \mathrm{ps},
$$

where $\Delta z$ is the distance between probes and $\varepsilon_{r}$ for Macor is about 6 . The sampling rate of 20 giga samples per second, or 50 ps between samples, permits only four sampling intervals between signals.

In Figure 17 the initial response of the D-dot probes is shown for cases where the test system broke down before the application of the UV laser pulse. For both cases the insulator is Macor at a -45 degree angle. In Shot \#869, Probe \#1 responded first, followed 100 ps (two sample intervals) later by Probe \#2 and 250 ps (five sample intervals) by Probe \#4. Timing was determined by position of the first "peak". This arrival sequence is consistent with a signal originating on the Probe \#1 side of the insulator near the anode triple junction and along a line that extends through both Probe \#1 and Probe \#4. In Shot 878 the signal arrived simultaneously at all three probes within the resolution of the oscilloscope. This could indicate a breakdown at the high voltage feed through to the test chamber.

This signal arrival sequence of Shot \#869 is seen on the shots where the flashover is initiated by the UV pulse illuminating the surface above Probe \#1. However, for the UV initiated flashover, it can be difficult to determine an initial signal front due to charging activity prior to flashover. Refer to Figure 13. Thus, the examples were chosen from non-UV breakdown shots to clearly show the time differences. The limited time resolution allows only rough estimates of the location of the breakdown/flashover events. It is still possible to verify which insulator face flashed over, i.e. the source of the signal. Figure 26 displays signals from two probes at the higher 40 Giga samples per second rate and illustrates the improved time resolution. 

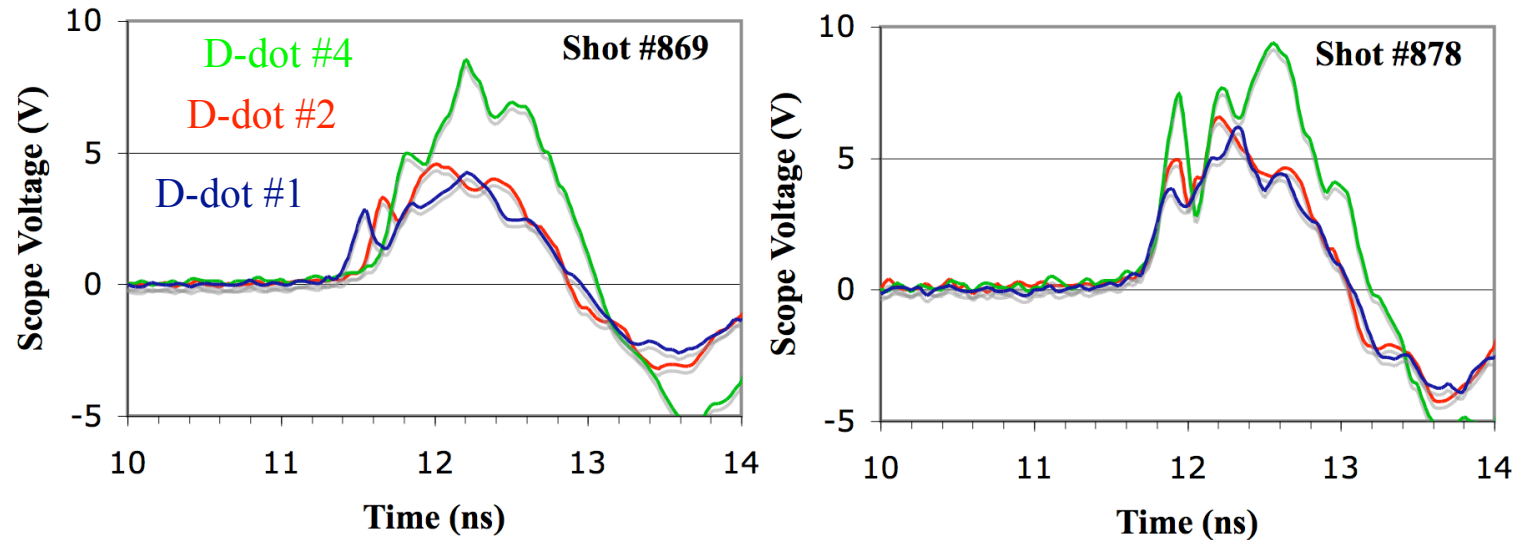

Figure 17. Arrival times of a signal at the different probes are emphasized in the plots. For Shot \#869 the signal originated near Probe \#1 while for Shot \#878 the origin was equidistance from all probes.

\section{Detecting Surface Charge}

The initial "bump" observed only on the Probe \#1 signal following the arrival of the UV laser pulse appears to be due to the charging of the insulator service. This bump can be seen in Figure 13. This effect is most apparent on the integrated signal. Figure 18 shows the integrated signals of Probes \#1 and \#2 after correcting for DC offsets. The integrated signals are approximately representative of the diode voltage. Refer to the previous section for details on converting to voltage. The important fact is that Probe \#1 indicates a change in diode voltage immediately after the start of UV illumination while the other probes do not. The D-dot probes actually respond to a change in the induced surface charge, $Q_{C}$ from Figure 1 . An assumption was made that the electric field due to the voltage across the diode was responsible for the induced charge on the probe.

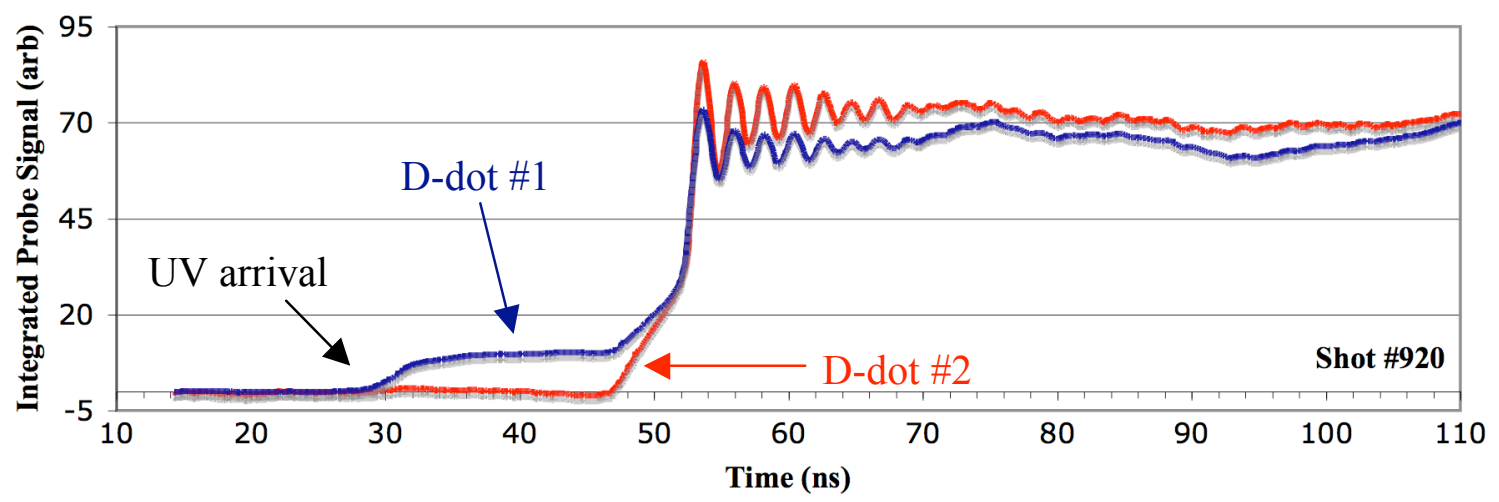

Figure 18. Plot of the integrated signals from D-dot \#1 and \#2.

Movement of electrons on the surface of the insulator could lead to sites of unbalanced charge resulting in fields that induce charge on the probes in addition to that of the charged electrodes. Especially for positive insulator angles, the enhanced fields at the anode triple junction (ATJ) would pull electrons ejected by the UV pulse away from the insulator. Assuming that the strength of the electric field falls off as the square of the distance, the field and induced charge at Probes $\# 2$ and $\# 3$ would be about $(2.7 \mathrm{~mm} / 20 \mathrm{~mm})^{2}=2 \%$ of 
that at Probe $\# 1$ for the +45 degree insulator angle. An order of magnitude estimate of the charge at the ATJ can be attempted. The electric field at the probe can be related to both the induced charge on the probe, $Q_{C}$, the charge at the ATJ, $q$ :, the radius of the probe, $r_{p}$, and the distance between the probe and the ATJ, $r$ :

$$
|E| \approx \frac{Q_{C}}{3 \pi \varepsilon r_{p}^{2}} \sim \frac{q}{4 \pi \varepsilon r^{2}} \Rightarrow q \sim Q_{C} \frac{r^{2}}{r_{p}^{2}} .
$$

Equations (2) and (5) can be used to show that the total charge induced on the probe is

$$
Q_{C}=\frac{1}{Z} \int V_{S} d t,
$$

where the integral is over the time span that $V_{S}$ is non-zero immediately following the UV pulse arrival. The vertical axis of the graph in Figure 18 is in units of pico coulombs (pC) when referring to charge. Thus the induced charge on the probe is about $10 \mathrm{pC}$. For $r=2.7 \mathrm{~mm}$ and $r_{p}=0.51 \mathrm{~mm}$,

$$
q \sim \frac{(2.7)^{2}}{(0.51)^{2}} 10 \mathrm{pC}=280 \mathrm{pC}
$$

Note that this is only a very crude estimate. Extensive modeling of the distribution of charge on the insulator surface and resulting electric field would be needed for an accurate calculation. This charging is also consistent with Lauer's ${ }^{3}$ proposed ATJ initiated breakdown mechanism.

The charging of, and movement of charge on, the insulator surface can also affect the shape of the probe response at flashover as shown in Figure 19. The raw oscilloscope signals of the three probes are shown with Probe \#4 on a different scale to accommodate a slightly different attenuator. In addition to the delays mentioned in the previous section, the shape of Probe \#1 for the initial peak is noticeably different than for the other probes. The raw data corresponds to a time rate of change of charge, or current.

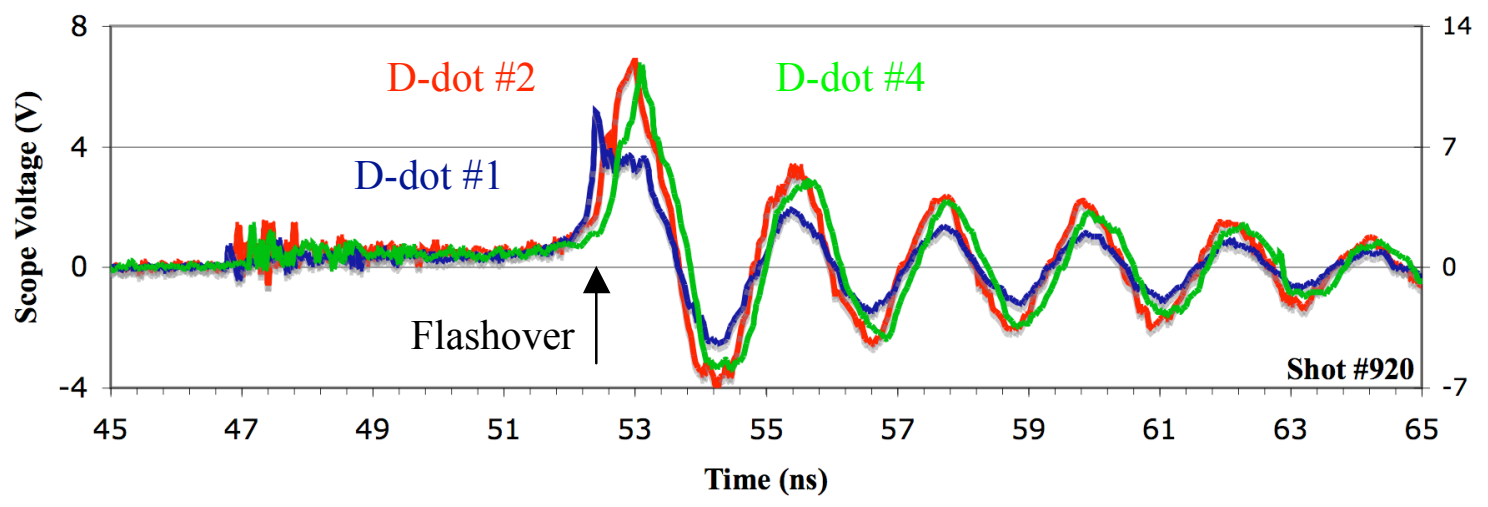

Figure 19. Expanded time scale of D-dot signals about the insulator flashover.

\section{Effect of Insulator Angle}

The effect of the insulator angle was mentioned above in regard to charging the insulator surface. Figure 20 is the counterpart of Figure 18 when the insulator is flipped over to produce a -45 degree angle. Probe \#1 is farther from the UV illuminated surface for this case and the enhanced electric field is at the cathode triple junction. Thus there is no noticeable effect on the probe signals with UV arrival. 


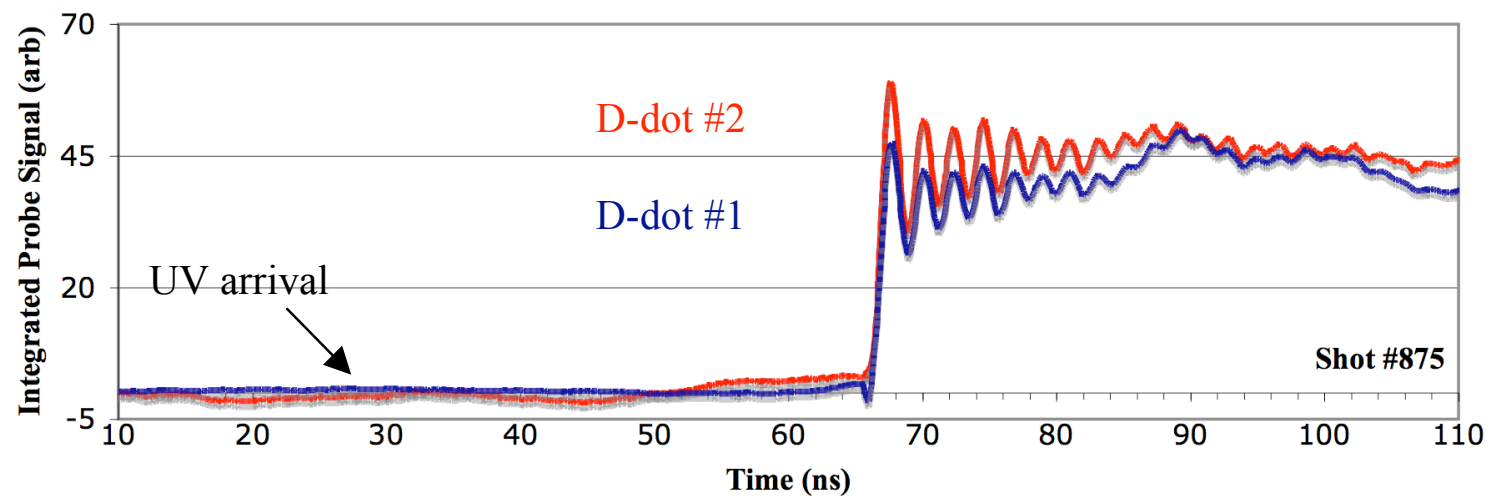

Figure 20. Plot of the integrated signals from D-dot \#1 and \#2 for a -45 degree insulator angle.

Of more interest is the probe response just prior to and during the insulator flashover. For positive angles, all the D-dot probes indicate a flow of charge crosses the diode gap loading (reducing) the diode voltage as shown in Figure 21. For negative angles, electrons coming from the vicinity of the CTJ will tend to strike the surface of the insulator. The negative dip just prior to flashover indicates electrons accumulating on the surface, possibly neutralizing a positive charge created with the arrival of the UV pulse.

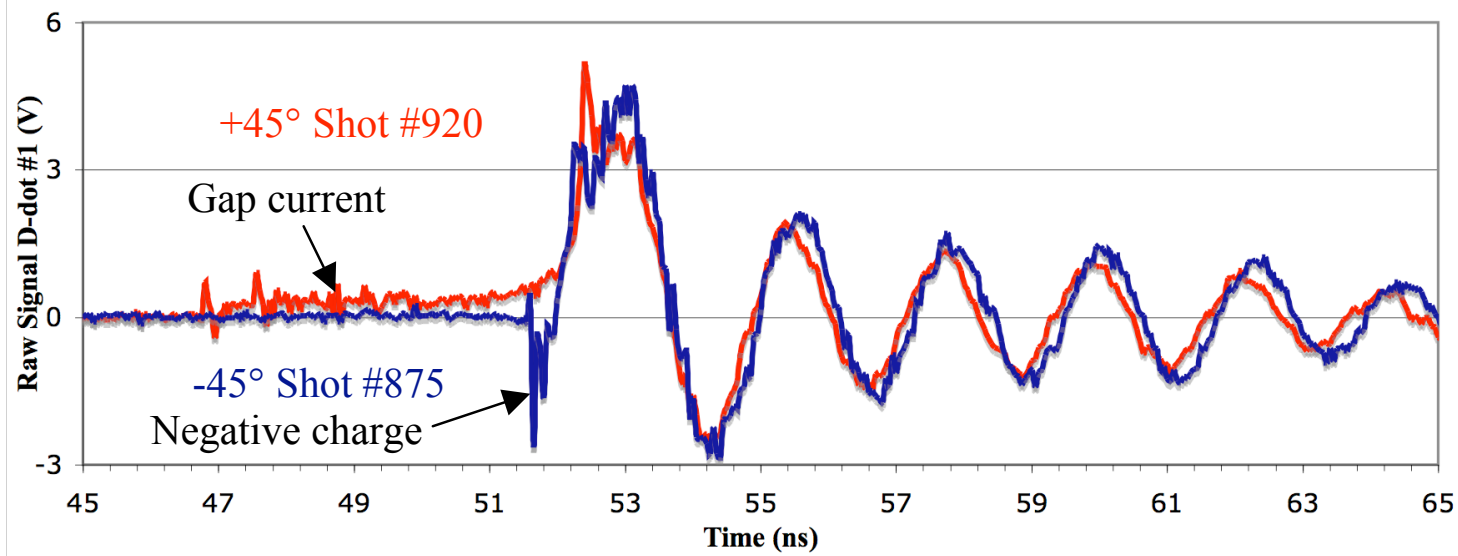

Figure 21. Expanded time scale of D-dot \#1 signals about the insulator flashover for $\pm 45^{\circ}$ insulator angles.

\section{Effect of Insulator Material}

The different responses of high density polyethylene (HDPE) and Macor are shown in Figures 22 and 23. For the shots shown, both insulators had +45 degree angles and the diodes were charged to $45 \mathrm{kV}$. Total UV energy was $31 \mathrm{~mJ}$ for Shot \#781 and $66 \mathrm{~mJ}$ for Shot \#920.

The response for HDPE at the arrival of UV was similar to that of Macor as shown in Figure 20. Detailed differences for the HDPE included a $\sim 2$ ns longer delay before charging was noted, charging for about $18 \mathrm{~ns}$ compared to $2 \mathrm{~ns}$ for the Macor, and a greater charge. The first two differences might be partly explained by the lower applied UV power. The greater charge might be due to the secondary electron emission characteristics of the two materials. 


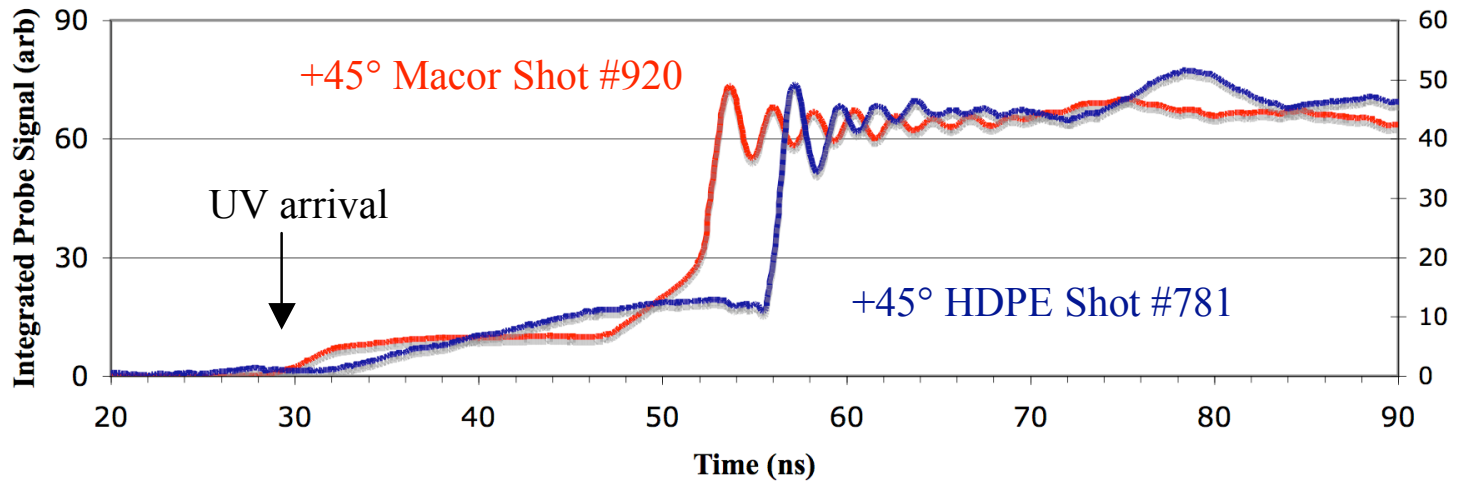

Figure 22. Plot of the integrated signals from D-dot \#1 for Macor and HDPE insulator.

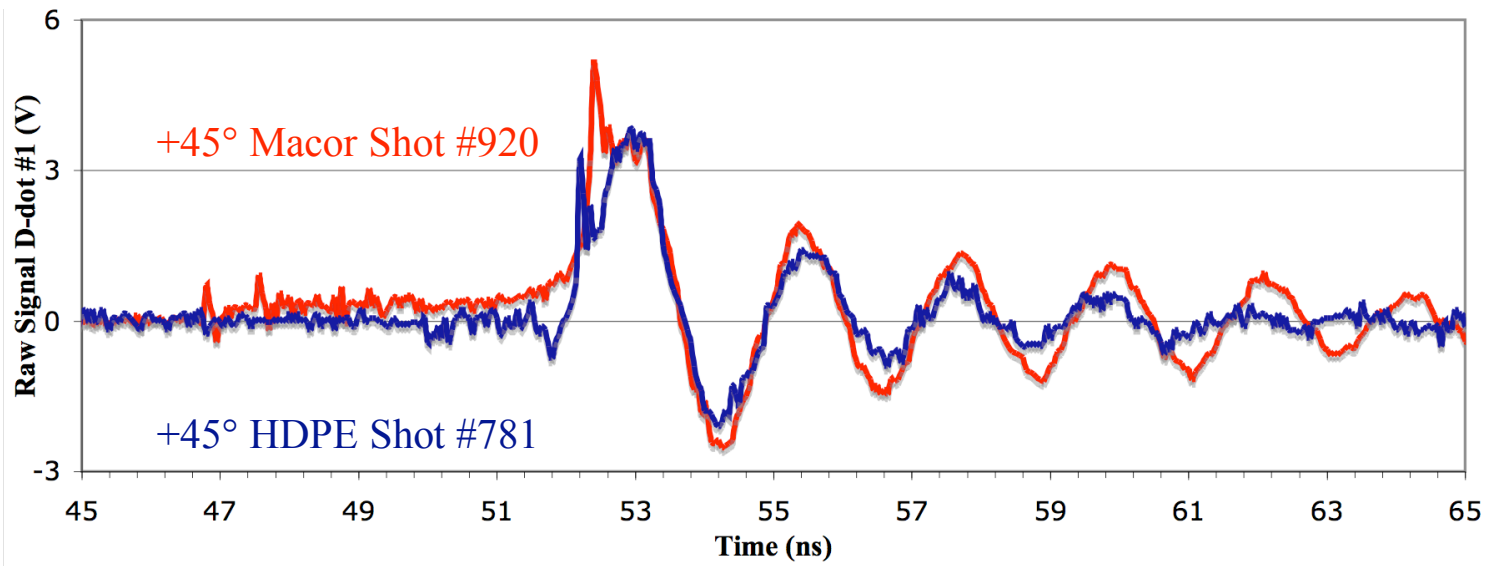

Figure 23. Expanded time scale of D-dot \#1 signals about the flashover for Macor and HDPE insulator.

The response of the two materials about flashover was noticeably different with the HDPE actually behaving somewhat like the Macor at -45 degrees with very little activity seen on Probe \#1 prior to a short period of negative charge accumulation followed immediately by flashover. Figure 24 shows that the other probes had signals similar to the +45 degree Macor, so the HDPE apparently charged differently near the ATJ than the Macor. Another difference was that the Macor showed charging activity for 5 ns prior to flashover compared to about $1 \mathrm{~ns}$ for the HDPE. This is particularly unexpected since the HDPE was exposed to a lower UV power. The timing sequence of the flashover at the three probes during Shot 781 was consistent with the flashover occurring on the insulator face over Probe \#1.

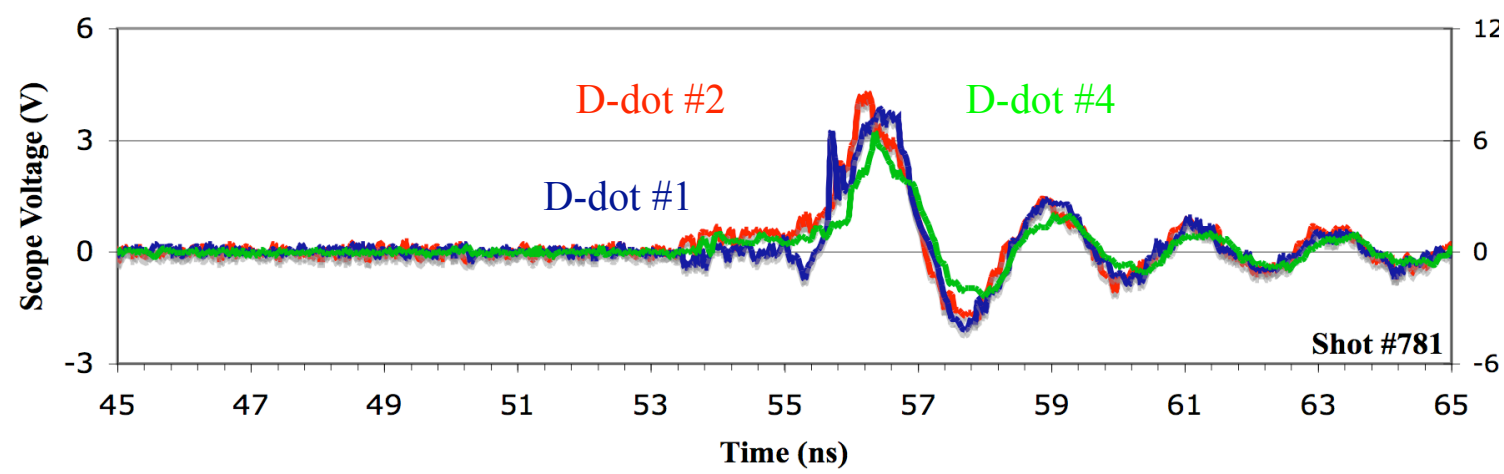

Figure 24. Expanded time scale of D-dot signals about the insulator flashover for HDPE. 


\section{Higher Resolution Probe Comparison}

A few flashovers were recorded at the 40 Giga sample/s time resolution for D-dot probes 1 and 4 . Figure 25 shows the probes signals from the UV pulse arrival, indicated by the pulse on probe 1, until about $20 \mathrm{~ns}$ after flashover. For this shot the insulator was Mycalex, a glass-mica machinable ceramic with $\varepsilon_{r}=6.8$, at +45 degrees, the electrodes were charged to $50 \mathrm{kV}$, and the laser pulse contained approximately $90 \mathrm{~mJ}$.

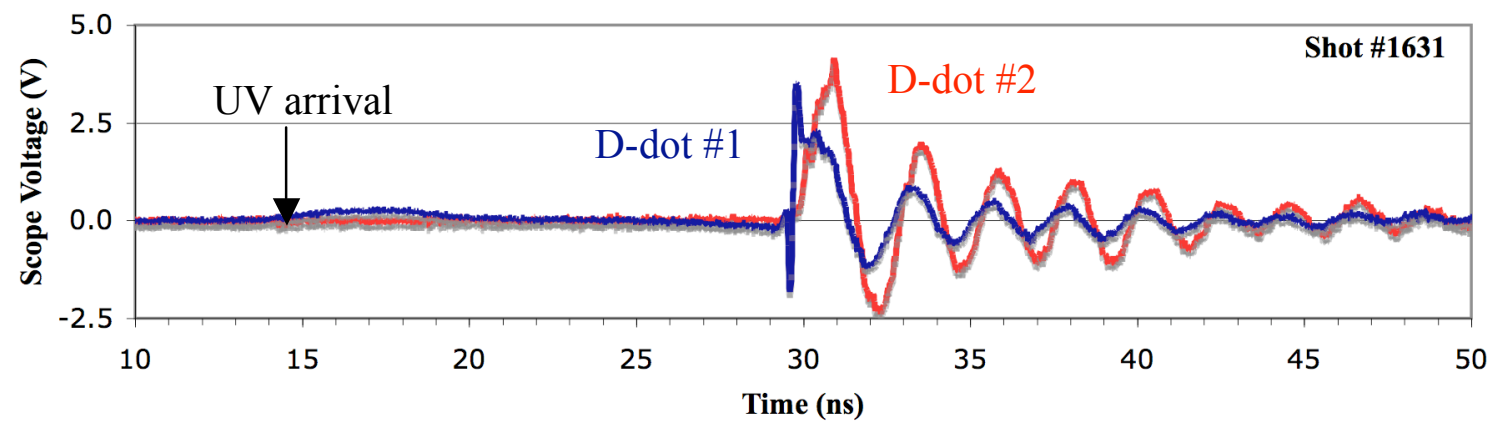

Figure 25. D-dot signals at 40 Giga samples/s about the insulator flashover for $+45^{\circ}$ Mycalex.

The expanded time scale of the probe signals shown in Figure 26 permits a number of interesting observations that support and complements the lower resolution data. The delay between the signal arriving at Probe \#1 and Probe \#4 is well resolved with $9 \pm 1$ time intervals of 25 ps. Starting at about $27 \mathrm{~ns}$, the positive charge accumulated on the surface as detected on Probe \#1 is lessened possibly by the movement of electrons along the surface towards the anode. The change in charge is more apparent in the integrated signal shown in Figure 27. This effect is similar to Shot \#781 in Figure 23. The decrease in the positive charge is reversed for about 100 ps followed by a relatively large drop that is in turn followed by a much greater increase. This variation is consistent with Enloe's ${ }^{4}$ description of a flashover where the initial sheet of charge flows across the surface effectively neutralizing any charge buildup followed by the charge sheet "lifing" away from the surface and presumably leaving a positive charge behind. For our system this evolution in surface charge occurs in less than $400 \mathrm{ps}$. After that time the collapsing electrode voltage would allow free electrons to return to the surface neutralizing any surface charge and the probe signals would be dominated by the LRC ringing of the test chamber. The phase difference in the ringing between Probe \#1 and \#4 could be related to their distances from the flashover/arc.

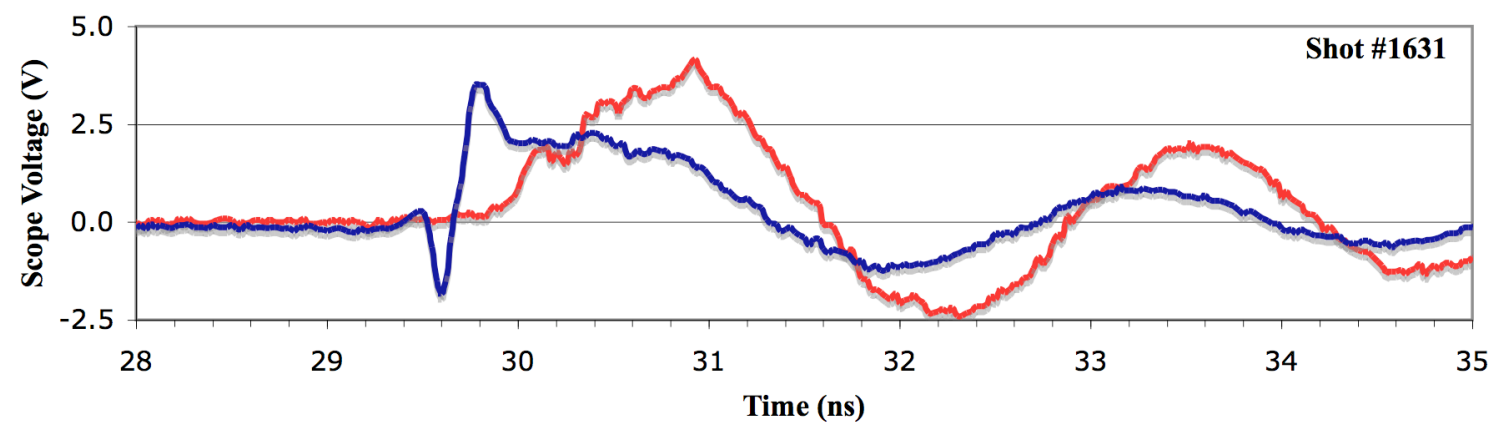

Figure 26. Expanded time scale of the D-dot signals shown in Figure 25. 


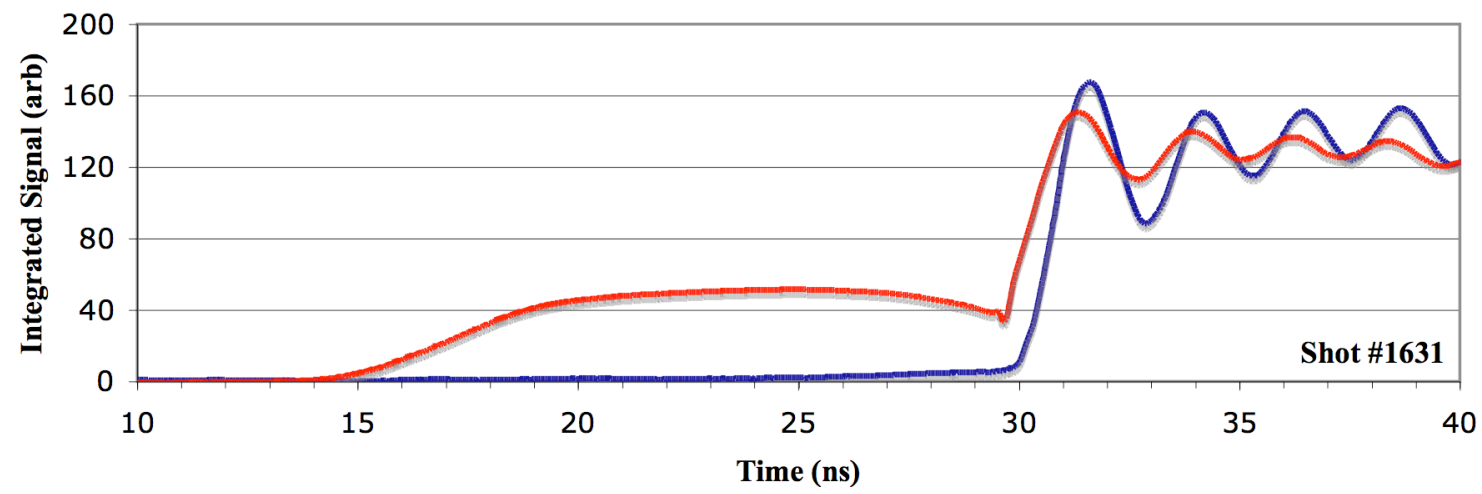

Figure 27. Integrated signals of the probes shown in Figure 25.

\section{Summary}

The D-dot probes demonstrated sufficient bandwidth and sensitivity to capture the electric fields associated with electrons moving across the face of a vacuum insulator during UV initiated flashover. The importance of using small probes that can be placed in the immediate vicinity of the flashover was emphasized by the difference in signals on adjacent probes separated by less than a $\mathrm{cm}$. The principle limitations on the probe performance were the bandwidth of the recording digitizer and difficulty of calibrating at frequencies above a few GHz. Of the two capacitances associated with probe performance, only the coupling to the electrode could be measured. The probes were relatively cheap to fabricate.

This was not a research project, but a demonstration of a technology to acquire data related to the mechanism(s) governing flashover of a vacuum insulator. The D-dot probes were successful at detecting the movement of charge and charge accumulation on the insulator surface. The probes could differentiate between the effects of insulator angle and material. The capability to locate the position of the flashover was marginal due to the sampling rate of the recording digitizer and number of recording channels, but sufficient to determine the side of the insulator involved. Determining the position of charges on the surface was even more limited due to the small signal. Placing more probes closer to the charge sites would only determine the position with faster digitizers. A more practical approach might be to increase the surface area of the probes to increase the signal strength.

The data acquired during the testing of the probes could be used in support of a research effort on vacuum insulator flashover. Although this demonstration was not meant to establish a mechanism for vacuum insulator flashover, some proposed mechanisms might be eliminated or refined by the data.

\section{Acknowledgements}

Greg Hawkins constructed the D-dot probes and installed them in the test chamber. Adam White and Emer Baluyot performed the airline calibration. Brian Kelly and Mike Shirk operated the UV Eximer laser during 
the insulator testing. Jim Crawford fabricated insulators and provided mechanical support. This work performed under the auspices of the U.S. Department of Energy by Lawrence Livermore National Laboratory under Contract DE-AC52-07NA27344 as an Enginering TechBase Project (TB-07-84).

\section{References}

${ }^{1}$ S. Humphries, Jr., Principles of Charged Particle Acceleration (Wiley, New York, 1985) pp. 275-276.

2 W.A. Stygar, et al., "D-Dot and B-Dot Montors for Z-Vacuum-Section Power-Flow Measurements," Pulsed Power Conf., Digest of Technical Papers, 1997, $11^{\text {th }}$ IEEE International, Vol. 2, pp. 1258-1263, June 1997

3 E.J. Lauer, "Electron Avalanche Model of Dielectric-Vacuum Surface Breakdown,” J. Appl. Phys. 102, 1133062007

${ }^{4}$ C.L. Enloe and R.M. Gilgenbach, "Spectroscopic Observations of UV-Laser-Induced Flashover Across an Angled Insulator," IEEE Trans. Plasma Sci., Vol. 17, No. 3, pp. 550-54, June 1989 


\section{Appendix A}

\section{List of Symbols with Definitions}

A: constant, used in derivations as an unknown to be determined

$\alpha$ : cable frequency dependent attenuation in $\mathrm{dB}$ per unit length, refer to equation (13)

$\beta$ : cable attenuation in $\mathrm{dB}$ per unit length, note that $\alpha=\beta \sqrt{\omega}$

B: constant, used in derivations as an unknown to be determined

$c$ : speed of light in vacuum

$C_{c}$ : coupling capacitance between the D-dot probe and the drive electrode

$C_{w}$ : coupling constant between the D-dot probe and the ground electrode (wall)

$d$ : gap spacing for parallel electrodes

$\vec{E}$ : electric field

$E_{\perp}$ : component of the electric field perpendicular to the surface

$E_{r}$ : radial component of the electric field

$\Delta$ : increment or small period of time

$\Delta z$ : increment of length, increment of length in the longitudinal (z) direction

$\varepsilon$ : electric permittivity

$\varepsilon_{o}$ : electric permittivity of vacuum

$\varepsilon_{r}:$ relative permittivity, $\varepsilon_{r}:=\varepsilon / \varepsilon_{o}$

I: current flowing through a circuit

$P$ : signal power

$P_{o}$ : reference signal power

$Q$ : surface charge

$\dot{Q}=\partial Q / \partial t$ : time rate of change of surface charge, equal to the current

$Q_{c}$ : surface charge induced on electrode

$Q_{w}$ : surface charge induced on conducting wall

$r$ : radius or radial distance

$r_{i}$ : radius of the inner conductor of a coaxial cable

$r_{o}$ : outer radius of the dielectric in a coaxial cable, same as the inner radius of the outer conductor

$R_{i}$ : radius of the inner conductor of a coaxial airline

$R_{o}$ : inner radius of the outer conductor of a coaxial airline

$\tau$. a time constant equal to $Z\left(C_{c}+C_{w}\right)$

$t^{\prime}$ : a dummy variable of integration. In this report it represents the time variation of a function

$V$ : voltage

$V_{o}$ : reference voltage

$<V>$ : rms value of oscillating voltage 
$\dot{V}=\partial V / \partial t$ : time rate of change of voltage, time derivative of voltage

$V_{D}$ : drive or applied voltage to the electrode, referenced with respect to ground. Note, when written as $V_{D}(t)$

it is a function of time, otherwise it is a constant unless specified as time dependent in the adjoining text.

$V_{S}$ : measured diagnostic voltage, always time dependent.

$\omega$ : frequency

$Z$ : impedance 


\section{Appendix B \\ Derivation of D-dot Probe Response to Specific $V_{D}$}

Two different drive voltages will be considered. The first is a constant linear ramp such that $\dot{V}_{D}$ is constant,

i.e.

$$
V_{D}(t)=V_{D}(0)+\dot{V}_{D} t
$$

Substituting $\tau$ for $Z\left(C_{c}+C_{w}\right)$ in Equation (6):

$$
\dot{V}_{S}(t)+\frac{1}{\tau} V_{S}(t)=\frac{Z C_{c}}{\tau} \dot{V}_{D}
$$

From inspection, the solution to Equation (B2) must have the form:

$$
V_{S}(t)=A\left(1-e^{-t / \tau}\right)
$$

where A is a constant to be determined. Substituting Equation (B3) into (B2):

$$
\begin{aligned}
\frac{A e^{-t / \tau}}{\tau}+\frac{A}{\tau}\left(1-e^{-t / \tau}\right) & =\frac{A}{\tau}=\frac{Z C_{c}}{\tau} \dot{V}_{D} \Rightarrow A=Z C_{c} \dot{V}_{D} \text {, and } \\
V_{S}(t) & =Z C_{c} \dot{V}_{D}\left(1-e^{-t / \tau}\right) .
\end{aligned}
$$

The second example is a constant amplitude and frequency oscillation:

$$
V_{D}(t)=V_{D} \sin (\omega t) \text {, and } \dot{V}_{D}(t)=\omega V_{D} \cos (\omega t) .
$$

Substituting Equation (B6) into Equation (B2):

$$
\dot{V}_{S}(t)+\frac{1}{\tau} V_{S}(t)=\frac{Z C_{c} \omega V_{D}}{\tau} \cos (\omega t)
$$

From inspection, the solution to Equation (B7) must have the form:

$$
V_{S}(t)=A \sin (\omega t)+B\left(\cos (\omega t)-e^{-t / \tau}\right) .
$$

Note that $V_{S}(0)=0$. Substituting Equation (B8) into (B7) and separating the different time dependencies:

$$
\begin{gathered}
e^{-t / \tau}\left\{\frac{B}{\tau}-\frac{B}{\tau}=0\right\} \\
\sin (\omega t)\left\{-B \omega+\frac{A}{\tau}=0\right\} \Rightarrow B=\frac{A}{\omega \tau} \\
\cos (\omega t)\left\{\omega A+\frac{B}{\tau}=\frac{Z C_{c} \omega V_{D}}{\tau}\right\}
\end{gathered}
$$

Substituting for $B$ in Equation (B11) and solving for $A$ :

$$
A=\frac{Z C_{c} \omega V_{D}}{\omega^{2} \tau^{2}+1} \omega \tau, \text { and } B=\frac{Z C_{c} \omega V_{D}}{\omega^{2} \tau^{2}+1} .
$$

Finally,

$$
V_{S}(t)=\frac{Z C_{c} \omega V_{D}}{\omega^{2} \tau^{2}+1}\left[\omega \tau \sin (\omega \tau)+\cos (\omega \tau)-e^{-t / \tau}\right] \text {. }
$$




\section{Appendix C}

\section{Nominal Specifications of Commercial 50 Ohm, Copper Jacket, Coaxial Cable}

Table 1: Nominal manufacturers specifications

\begin{tabular}{|c|c|c|c|c|}
\hline Designation $^{1}$ & $\begin{array}{c}\text { Inner Conductor } \\
\text { Diameter (in./mm) }\end{array}$ & $\begin{array}{c}\text { Dielectric }^{2} \\
\text { OD (in./mm) }\end{array}$ & $\begin{array}{c}\text { Outer Conductor } \\
\text { OD (in./mm) }\end{array}$ & $\begin{array}{c}\text { Attenuation per } 100 \mathrm{ft} \\
1 / 5 / 10 \mathrm{GHz}\end{array}$ \\
\hline RG401/U & $0.065 / 1.651$ & $0.215 / 5.461$ & $0.250 / 6.350$ & $9.7 \mathrm{~dB} / 26.1 \mathrm{~dB} / 40.7 \mathrm{~dB}$ \\
\hline RG402/U & $0.036 / 0.914$ & $0.119 / 3.023$ & $0.141 / 3.581$ & $13 \mathrm{~dB} / 36 \mathrm{~dB} / 62 \mathrm{~dB}$ \\
\hline PE-118SR & $0.032 / 0.813$ & $0.095 / 2.413^{3}$ & $0.118 / 2.997$ & $12.2 \mathrm{~dB} / 28.7 \mathrm{~dB} / 42.1 \mathrm{~dB}$ \\
\hline RG405/U & $0.0201 / 0.510$ & $0.066 / 1.676$ & $0.085 / 2.159$ & $22 \mathrm{~dB} / 50 \mathrm{~dB} / 80 \mathrm{~dB}$ \\
\hline PE-047SR & $0.0113 / 0.287$ & $0.037 / 0.940$ & $0.047 / 1.194$ & $40 \mathrm{~dB} / 105 \mathrm{~dB} / 130 \mathrm{~dB}$ \\
\hline PE-034SR & $0.008 / 0.203$ & $0.026 / 0.660$ & $0.034 / 0.864$ & $60 \mathrm{~dB} / 120 \mathrm{~dB} / 190 \mathrm{~dB}$ \\
\hline PE-020SR & $0.0044 / 0.112$ & $0.015 / 0.381$ & $0.020 / 0.508$ & $85 \mathrm{~dB} / 175 \mathrm{~dB} / 280 \mathrm{~dB}$ \\
\hline
\end{tabular}

Notes: ${ }^{1}$ Pasternack Enterprises Designations - the use here does not imply endorsement of their cable

${ }^{2}$ The dielectric is polytetrafluoroethylene with a $\varepsilon_{r}$ of approximately 2.1 , unless otherwise noted

${ }^{3}$ The dielectric is low density polytetrafluoroethylene with a $\varepsilon_{r}$ of approximately 1.7

Table 2: Nominal capacitances and time constants based on a $1 \mathrm{~cm}$ spaced parallel plate geometry

\begin{tabular}{|c|c|c|c|c|c|c|c|c|}
\hline & \multicolumn{4}{|c|}{ Vacuum } & \multicolumn{4}{c|}{ Insulator $\left(\varepsilon_{r}=2.3\right)$} \\
\hline Designation & $C_{C}(\mathrm{fF})$ & $C_{W}(\mathrm{fF})$ & $C_{W} / C_{C}$ & $\tau(\mathrm{ps})$ & $C_{C}(\mathrm{fF})$ & $C_{W}(\mathrm{fF})$ & $C_{W} / C_{C}$ & $\tau(\mathrm{ps})$ \\
\hline RG401/U & 7.21 & 37.4 & 5.2 & 2.23 & 15.53 & 74.6 & 4.8 & 4.51 \\
\hline RG402/U & 2.185 & 23.2 & 10.6 & 1.27 & 4.669 & 44.8 & 9.6 & 2.47 \\
\hline PE-118SR & 1.481 & 10.2 & 6.9 & 0.584 & 3.244 & 30.0 & 9.2 & 1.66 \\
\hline RG405/U & 0.663 & 13.4 & 20.2 & 0.703 & 1.413 & 25.7 & 18.2 & 1.36 \\
\hline PE-047SR & 0.206 & 8.5 & 41.3 & 0.435 & 0.434 & 15.2 & 35.0 & 0.782 \\
\hline PE-034SR & 0.0996 & 5.7 & 57.2 & 0.290 & 0.208 & 10.2 & 49.0 & 0.520 \\
\hline
\end{tabular}




\section{Appendix D \\ Suggested Design Estimators}

For a Parallel Plate Geometry the coupling capacitance can be expressed as:

$$
C_{c}=\frac{Q}{V}=\frac{\varepsilon E_{\perp} \text { Area }}{\mathrm{V}}=\frac{\varepsilon \text { Area }}{d}=\frac{\varepsilon_{r} \varepsilon_{o} \pi r^{2}}{d}
$$

The derivation of Equation (D-1) assumes that there is no distortion to the electric field over the probe tip from the field for infinite parallel plates, i.e. $|\vec{E}|=E_{\perp}=V / d$, and only the area of the top of the probe contributes. $V$ is the potential difference between the plates separated by a distance $d$. The electric field, as illustrated in Figure 5, is not uniform on the probe tip and extends for some distance along the side of the probe. To compensate for these effects, the area term can be increased, e.g.

$$
\begin{gathered}
\text { Area }=\pi r_{i}^{2}+2 \pi r_{i}\left(\frac{r_{o}-r_{i}}{2}\right)=\pi r_{i} r_{o}, \text { or } \\
C_{c}=\frac{\varepsilon_{r} \varepsilon_{o} \pi r_{i} r_{o}}{d} .
\end{gathered}
$$

Equation (D-3) is good to about $5 \%$ to $10 \%$ for the geometries of interest. Note that for 50 ohm cable, this estimate is about 3 times larger than if the sides were neglected, $\frac{r_{o}}{r_{i}} \approx 3$.

For a Coaxial Geometry with the probe installed in the outer wall, Equation (D-2) is appropriate for the area. The electric field is taken as that for an undisturbed coaxial cable:

$$
\begin{gathered}
|\vec{E}|=E_{\perp}=E_{r}=V / R_{o} \ln \left(R_{o} / R_{i}\right), \text { or } \\
C_{c}=\frac{Q}{V}=\frac{\varepsilon E_{\perp} \text { Area }}{\mathrm{V}}=\frac{\varepsilon \text { Area }}{R_{o} \ln \left(R_{o} / R_{i}\right)}=\frac{\varepsilon_{r} \varepsilon_{o} \pi r_{i} r_{o}}{R_{o} \ln \left(R_{o} / R_{i}\right)} .
\end{gathered}
$$

The coupling of the probe to the wall is the same for either geometry. The following expression is based on a fit to the calculated values given in Appendix $\mathrm{C}$ and provides a very rough estimate:

$$
C_{w}=\frac{\varepsilon_{r} \varepsilon_{o} 8 \pi\left(r_{o}-r_{i}\right)}{\ln \left(r_{o} / r_{i}\right)}
$$

